\title{
Comparative Study of Single-Atom Gold and Iridium on $\mathrm{CeO}_{2}\{111\}$
}

\author{
Cameron J. Owen ${ }^{a)}$ and Stephen J. Jenkins ${ }^{\text {b) }}$ \\ Yusuf Hamied Department of Chemistry, University of Cambridge, Lensfield Road, Cambridge, CB2 1EW, \\ United Kingdom
}

(Dated: 31 March 2021)

Oxide-supported single-atom catalysts have shown promise for a variety of heterogeneous processes. In addition to their inherent activity and selectivity, these materials come at much lower financial cost, avoiding the use of full-bodied precious-metal catalysts, but at the conceptual expense that more complex structural and electronic considerations need to be understood if we are to exploit their full potential. Here, we focus upon the adsorption of single-atom iridium at both stoichiometric and defective $\mathrm{CeO}_{2}\{111\}$ surfaces, by means of first-principles Density Functional Theory (DFT). Reference calculations for the adsorption of single-atom gold, on the same set of substrates, provide a valuable set of benchmarks against which to interpret our iridium results.

\section{INTRODUCTION}

Single-atom catalysts (SACs) are a rather new class of material showing great potential for a variety of catalytic applications. Until relatively recently, it was thought that SACs would be too thermodynamically unstable for practical use, being presumed prone to sintering at temperatures typically relevant for heterogeneous catalysis. Work reported over the last decade has, however, cast this assumption into doubt, especially in regard to isolated precious-metal adatoms on metal-oxide supports. ${ }^{1,2}$ Indeed, it has been shown that several SACs (e.g. $\mathrm{Pt}_{1} / \mathrm{FeO}_{x},{ }^{3} \mathrm{Au}_{1} / \mathrm{CeO}_{2},{ }^{4-15} \mathrm{Ir}_{1} / \mathrm{CeO}_{2},{ }^{16}$ etc.) are thermodynamically stable under plausible reaction conditions, and that the active precious-metal adatoms neither aggregate into larger nanoparticles nor diffuse into the underlying oxide. For the gold and platinum systems, this finding was also shown to be robust after exposure of the SACs to gas-phase reactant molecules. Such an observation is vitally important, as it has previously been demonstrated that surface alloys and nanoparticle assemblies can undergo dynamic rearrangement, not only as a function of temperature but also as a function of reactant gas pressure. ${ }^{17}$

Despite intense experimental and computational investigation focussing upon the thermodynamic stability of SACs, much remains to be understood in relation to their inherent activity for a variety of catalytic processes. In particular, the geometries of active sites in operando are subject to ongoing debate, in large part due to limitations of the available experimental methods. Current techniques, such as Scanning Transmission Electron Microscopy (STEM), X-Ray Absorption Near-Edge Structure (XANES), Scanning Tunnelling Microscopy (STM), Reflection Absorption Infrared Spectroscopy (RAIRS), and Temperature Programmed Desorption (TPD) permit the characterisation of surfaces as a whole, but yield limited (or no) information regarding active site geometries and mechanisms on the sub-nanometre length scale. Specifically, STEM data can confirm that preciousmetal adatoms are dispersed on a given metal-oxide support,

\footnotetext{
a) Also at Department of Chemistry and Chemical Biology, Harvard University, 12 Oxford Street, Cambridge, MA, USA 02138

${ }^{b)}$ Electronic mail: sjj24@cam.ac.uk
}

but lighter atoms (e.g. oxygen) cannot be imaged with sufficient resolution to permit detailed experimental determination of localised binding geometry. ${ }^{18}$ This is indeed an unfortunate limitation, since both XANES data and first-principles Density Functional Theory (DFT) calculations suggest that precious-metal adatoms may preferentially coordinate to substrate oxygen atoms. ${ }^{16,19}$ Speaking of XANES, the coordination number of precious-metal adatoms can be determined by this method, allowing for inferences to be drawn regarding the localised binding geometry, but reliable data is entirely dependent upon homogeneity in the sample. ${ }^{19}$ These experimental limitations underline the importance of high-quality DFT calculations to provide essential geometric information for active SACs, especially for characterisation of preciousmetal adatom binding sites. ${ }^{13}$

One particularly interesting aspect of SACs is that the lowcoordination environment of precious-metal adatoms on oxide supports may be thought of as analogous to metal centres coordinated to surface-bound ligands, and indeed the deposition of coordination complexes onto suitable surfaces may well provide an alternative viable route for the fabrication of stable SACs, perhaps even replicating the local environments found in existing precious-metal homogeneous catalysts. ${ }^{19}$ Much recent attention has, therefore, been centred on the use of SACs to bridge between the domains of heterogeneous and homogeneous catalysis, whereby long-established design principles from the latter discipline could be translated to the former, with a view towards boosting efficiency through operation under continuous flow conditions.

Notwithstanding these exciting prospects, however, the most prosaic motivation for the development of SACs remains the efficiency gain associated with reducing the number of catalytically irrelevant precious-metal atoms that must be deployed to achieve a given reaction. Full-bodied preciousmetal catalysts, in which only those atoms presenting at the surface are active in heterogeneous reactions, are inevitably wasteful of their most expensive resource - the precious-metal atoms themselves. In a SAC, by way of contrast, atom utilisation of the catalytically active metal can potentially approach $100 \%$, and similar overall catalytic performance may be achieved at a fraction of the financial cost. ${ }^{20}$ Our chosen systems for the present study exemplify this strategy perfectly, with the cost per mole of gold or iridium atoms typically ex- 
ceeding that of cerium atoms (in oxide form) by a factor of around 200.

Several $d$ - and $f$-block oxide supports have been employed in a variety of SACs, but not all are able to effectively bind individual metal adatoms and to maintain isolation of the same. Most importantly, the propensity of precious-metal adatoms toward sintering needs to be low, especially over the temperature range relevant to the intended catalytic application, but since isolated precious-metal adatoms are likely to exhibit both high mobility and high potential energy this may seem to be a forlorn hope. Nevertheless, certain single-atom/metaloxide pairs have shown marked resistance to sintering, enabling increased reactivity and longer catalytic lifetimes. ${ }^{21}$ Another important consideration is resistance toward diffusion of precious-metal adatoms into the support material itself, which is dictated by the strength of surface binding relative to the exothermicity of metal-oxide formation. ${ }^{16,22}$

Amongst potential oxide supports, cerium dioxide (ceria) has been shown to stabilize individual metal adatoms on its $\{111\}$ surface. ${ }^{1,2}$ The specific surface energy of this particular $\mathrm{CeO}_{2}$ facet was previously calculated to be $0.33 \mathrm{eV} . \AA^{-2}$ and $0.73 \mathrm{eV} . \AA^{-2}$ more stable than the $\{110\}$ and $\{100\}$ facets, respectively, ${ }^{23}$ and on this basis we focus upon the $\{111\}$ surface exclusively in the present work. In addition to the dioxide, studied here, which adopts the cubic fluorite-type structure $(\mathrm{Fm} 3 \mathrm{~m})$ cerium also forms a sesquioxide, $\mathrm{Ce}_{2} \mathrm{O}_{3}$, which adopts a hexagonal structure $(P 3 m 1)$. The stability of the latter form suggests that ceria is likely to be easily reducible, and that its surface will exhibit a substantial density of oxygen vacancy defects, especially at high temperatures in the absence of an oxidising atmosphere. Despite the ideal bulk dioxide's wide band gap, determined experimentally ${ }^{24-27}$ to be $3.2 \mathrm{eV}$, the surface may therefore be expected to display quite interesting electronic features. Moreover, the stoichiometric surface is expected to be an excellent oxygen donor, ${ }^{13}$ which may be of great relevance to reactions taking place via the Mars-van Krevelen mechanism. ${ }^{28}$ Similarly, the stoichiometric surface ought to be a strong electron acceptor, implying the likelihood of substantial charge transfer upon the adsorption of metallic adspecies. This may lead to an increase in the catalytic ability of isolated adatoms, analogous to changes of a central atom's oxidation state in the field of homogeneous catalysis. ${ }^{19}$

Regarding its structure, the stoichiometric $\mathrm{CeO}_{2}\{111\}$ surface is terminated by a layer containing seven-fold coordinated cerium cations (oxidation number +4 ) and twice as many three-fold coordinated oxygen anions (oxidation number -2). Upon abstraction of a neutral oxygen atom, however, two "spare" electrons must be accommodated in the vicinity of the defect, and these are widely believed to localise on the Ce $4 f$ orbitals belonging to two of the three vacancy-adjacent cerium ions, reducing them to the +3 oxidation state..$^{29,30}$ The correct description of this electron localisation (as opposed to delocalisation of charge around all three neighbouring cerium ions) is a challenge for DFT calculations, hinging upon an appropriate treatment of strong correlation (see discussion in the Methodology section). Furthermore, despite the fact that oxygen vacancies are known to cluster on the surface at high concentrations, ${ }^{31-34}$ we note that STM experiments reported by Esch $e t a l^{33}$ confirm the dominance of isolated vacancies under moderate reducing conditions.

Turning now to the adsorption of precious-metal adatoms, gold on ceria has attracted sustained attention due to its excellent activity in the water-gas shift (WGS) reaction. ${ }^{4,6,13}$ In the early years of the present century, some effort was expended towards understanding the efficiency of ceria-supported gold in this regard. The elegant experimental work of FlytzaniStephanopoulos and co-workers ${ }^{4}$ demonstrated clearly that activity was retained even once all gold content bar the very smallest clusters (most likely single adatoms) had been leached from the catalyst. Liu et $a l^{6}$ subsequently rationalised this, on the basis of first-principles DFT, in terms of the partial positive charge acquired by individual gold adatoms on the stoichiometric surface. They further showed that a partial positive charge was also present on the outer atoms of $\mathrm{Au}_{4}$ clusters that were anchored into an oxygen vacancy via a central negatively charged gold atom, and presented a mechanistic pathway for the WGS reaction in which these formed the active sites. Zhang et $a l^{12}$ subsequently confirmed that a positive partial charge was present amongst the peripheral atoms of clusters up to $\mathrm{Au}_{11}$ that were similarly anchored. Tibiletti et $a l,{ }^{5}$ on the other hand, found via similar methods that gold preferentially resides at cerium vacancies, should these be present at the surface, and that larger clusters may indeed be bound to the surface via nucleation at these sites too. To further test the activity of gold on ceria beyond the WGS reaction, Shapovalov $e a^{7}$ employed DFT to study CO

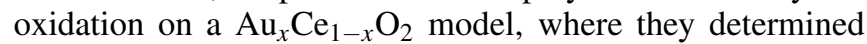
that gold can both facilitate oxygen vacancy formation and the subsequent evolution of carbon dioxide. Outside of the realm of surface science, gold monomers and cationic dimers have attracted a degree of attention for the activation of methane in the gas phase, ${ }^{35,36}$ and we therefore hypothesise here that dispersed single-atom gold on $\mathrm{CeO}_{2}\{111\}$ may potentially also provide catalytic centres for hydrocarbon activation.

Comparatively, the adsorption of iridium on ceria has received far less attention than that of gold. Recently, however, O'Connor et $a l^{16}$ predicted, on the basis of DFT calculations, that individual iridium adatoms should bind exothermically on stoichiometric $\mathrm{CeO}_{2}\{111\}$ in hollow sites formed by three adjacent surface oxygen atoms. The adatoms were further predicted to acquire a partial positive charge, raising the possibility that single-atom iridium might be especially active in catalysing heterogeneous oxidation reactions. Indeed, $\operatorname{IrO}_{2}\{110\}$ has recently shown promise in the low-temperature $(150 \mathrm{~K})$ activation of methane, ${ }^{37}$ so it is tempting to speculate that similar activity might possibly be achieved by single-atom iridium supported on $\mathrm{CeO}_{2}\{111\}$. As a first step, however, it will be necessary to characterise the structure and electronic properties of such adatoms, not only on the stoichiometric surface, but also on defective surfaces. The purpose of the present work is to explore just these aspects of the $\operatorname{Ir}_{1} / \mathrm{CeO}_{2}\{111\}$ system, with particular reference to comparison with the more familiar $\mathrm{Au}_{1} / \mathrm{CeO}_{2}\{111\}$ system. 


\section{METHODOLOGY}

The present work was carried out using the CASTEP computer code, ${ }^{38}$ which implements first-principles DFT subject to periodic boundary conditions. Calculations were performed within a supercell of lateral dimensions equivalent to a $(2 \times 2)$ surface unit cell (consistent with the experimental bulk lattice constant of $5.41 \AA$ ) and surface-normal length equivalent to twelve O-Ce-O trilayers (Fig. 1). Electronic wavefunctions were represented within a plane-wave basis set, truncated at a cutoff energy of $500 \mathrm{eV}$, and the Brillouin zone was sampled on a $2 \times 2 \times 1$ Monkhorst-Pack k-point mesh. ${ }^{39}$ Energy minimisation of the electronic degrees of freedom was accomplished within the ensemble DFT scheme of Marzari et $a l .{ }^{40}$ Twelve valence electrons were included for $\mathrm{Ce}\left(5 s^{2}, 5 p^{6}\right.$, $\left.6 s^{2}, 5 d^{1}, 4 f^{1}\right)$ while six were considered for $\mathrm{O}\left(2 s^{2}, 2 p^{4}\right)$. For $\mathrm{Au}$, we treated 33 valence electrons explicitly $\left(5 s^{2}, 5 p^{6}, 4 f^{14}\right.$, $\left.6 s^{1}, 5 d^{10}\right)$ and for Ir we included $31\left(5 s^{2}, 5 p^{6}, 4 f^{14}, 6 s^{2}\right.$, $\left.5 d^{7}\right)$. All other electrons were treated implicitly within the ionic cores, while core-valence interactions were incorporated via ultrasoft pseudopotentials ${ }^{41}$ calculated by CASTEP's onthe-fly algorithm. Exchange and correlation interactions were included through the spin-polarised PBE functional, ${ }^{42}$ augmented by a Hubbard term (see below). Although the commonly employed RPBE functional generally performs better than PBE in reproducing experimental adsorption energies, we note that even its originators considered it would likely be less accurate than the PBE functional in calculating certain structural properties ${ }^{43}$ moreover, it is not at all clear that any perceived advantage of the RPBE functional would actually carry over to cases where the Hubbard term is included, so we have opted to persist with the earlier functional for the present work. Surface structures were modelled, subject to the parameters described above, by means of slabs comprising five O-Ce-O trilayers (Fig. 1) with three trilayers on the side representing the surface permitted to relax in response to the calculated forces (according to the BFGS algorithm ${ }^{44}$ ).

One potential drawback with the use of DFT is that it is known to fail in systems where electron correlation is particularly strong. Standard exchange-correlation functionals, based on a mean-field approximation, are simply unable to capture the dynamic contributions that can dominate electronic behaviour when available states are highly localised - a deficiency often manifest in the erroneous distribution of electron density between multiple such states, when a more accurate treatment would reveal occupancy of only a small subset. Ceria, in which the highly localised $4 f$-states of cerium play an important role, is certainly a case in point, and it is therefore necessary to adapt the standard DFT methodology to account for this fact. The usual (and most straightforward) solution is to incorporate a so-called Hubbard term in the Hamiltonian, generally denoted $U$, that energetically penalises the presence of two electrons within a single orbital. The magnitude of the $U$ term is, unfortunately, not itself calculable within the framework of DFT, and so must somehow be estimated.

Fortunately, ceria surfaces have been extensively studied via this approach, and the literature provides a fairly clear steer that $U$ values (applied to the cerium $4 f$ electrons) in
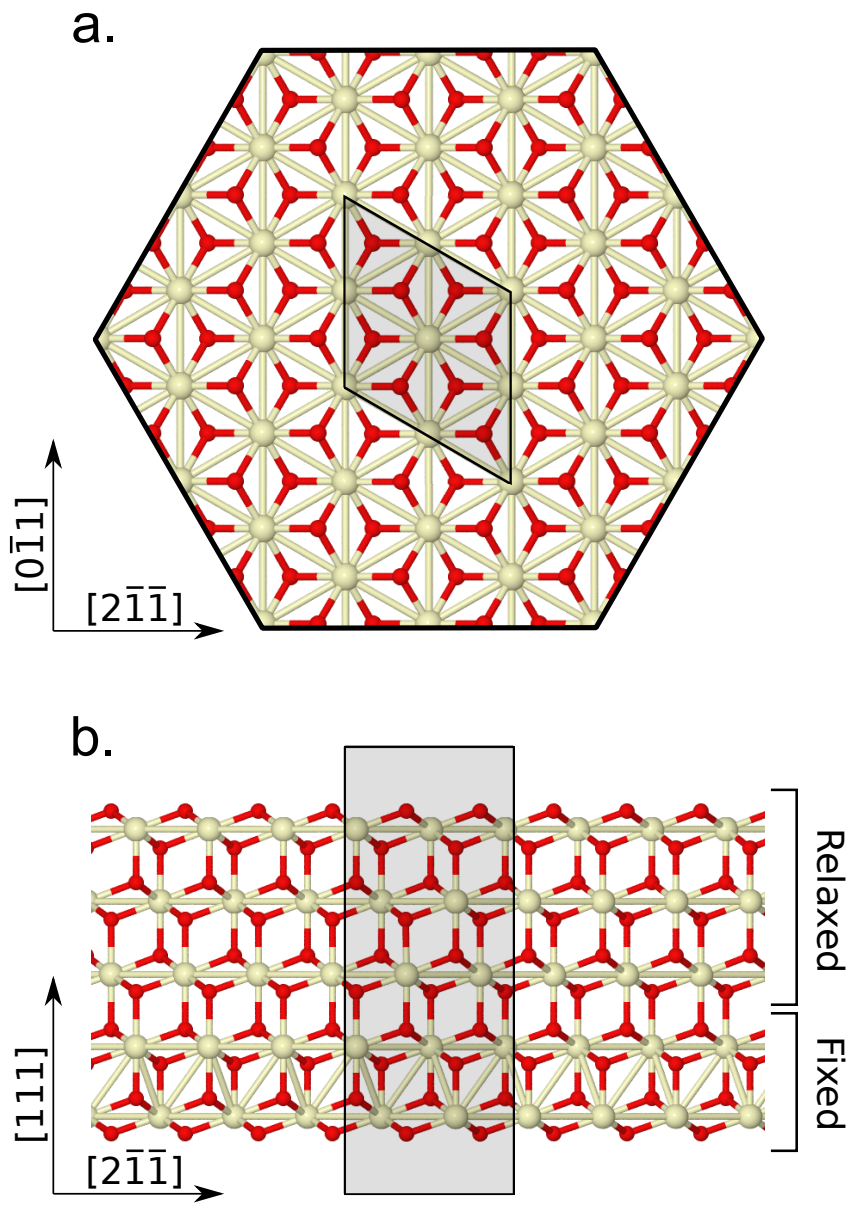

FIG. 1. Orthographic top-down and side views of the $\mathrm{CeO}_{2}\{111\}$ surface. In (a) the top-down view depicts only the uppermost layer, for reasons of clarity, with cerium depicted by grey spheres and oxygen by red ones; a single $(2 \times 2)$ unit cell, as used in the present calculations, is shown shaded. In (b) the side view shows the vertical extent of the slab, with fixed and relaxed layers indicated; the supercell employed in the present calculations is twice the length of the shaded region, with the additional volume comprising nothing but vacuum.

the range $1.5-6.0 \mathrm{eV}$ are likely to produce physically reasonable results; moreover, values close to $5.0 \mathrm{eV}$ have been most often employed alongside Generalised Gradient Approximation (GGA) functionals, of which the PBE functional used here is an example. ${ }^{13}$ That said, the precise value needed may vary quite legitimately between different works, depending not only upon details of the Hubbard scheme's implementation, but also upon the exchange-correlation functional and/or pseudopotentials used. An important benchmark, in the context of the present system, is that the calculation resulting from a particular choice for $U$ must be able to reproduce appropriately both the formation energy of a surface oxygen vacancy and the consequent localisation of electrons on just two of the three neighbouring cerium atoms.

In the present work, we first established that proper localisation of electrons in such circumstances was achieved once 
$U$ exceeded a value of $3 \mathrm{eV}$, but noted that the computed formation energy decreased strongly as this parameter was further increased. Indeed, setting $U$ precisely equal to $3 \mathrm{eV}$ gave the best agreement with our prior calculations, ${ }^{8,11-13,34}$ not only for the oxygen vacancy formation energy (that of the cerium vacancy diverges a little more, albeit remaining within an acceptable margin of c.10\%) but also for the adsorption energy of a gold adatom on the stoichiometric surface, and we have therefore adopted that value for all the calculations reported here (see Supporting Information for further details). Loschen $e t a l^{45}$ have previously argued that such a value represents a good choice when using the PW91 exchangecorrelation functional, ${ }^{46}$ which is similar to the PBE functional employed here. We emphasise, nonetheless, that there is no uniquely superior value to be determined for this parameter. Even values derived from first-principles calculations are really only justified on the grounds of self-consistency, rather than any rigorous formal footing. All one can demand is that physically reasonable results are obtained from one's choice, and that comparisons between different studies are assessed with due caution. This consideration lies behind our decision here to extensively describe results for the somewhat familiar $\mathrm{Au}_{1} / \mathrm{CeO}_{2}\{111\}$ system alongside those for the more novel $\mathrm{Ir}_{1} / \mathrm{CeO}_{2}\{111\}$ system, in order that our findings for iridium could be more readily compared with those for gold.

In the analysis that follows, extensive use will be made of projected density of states (PDOS) plots, in which the eigenfunctions calculated within the plane-wave basis are projected onto localised atomic orbitals calculated during generation of the pseudopotentials. The same orbitals are also used for Mulliken population analysis, ${ }^{47}$ and include all the valence states listed for each species in the first paragraph of this section. In general, it is important to carefully consider which states to include in both PDOS and Mulliken schemes, since low-lying states that are unoccupied in the ground-state configuration of an isolated atom may become incorporated into occupied states upon interaction with other atoms. For this reason, we considered it prudent to include $\mathrm{Ce} 6 p$ projectors, achieving charge spilling parameters below $0.15 \%$ in all systems studied. Inclusion of additional projectors ought always to be approached cautiously, however, given the risk of overfitting presented by the non-orthogonality of orbitals located on different atoms.

Finally, it will be helpful here to clarify some of our definitions regarding the energetics of our surfaces. Assuming a process occurring at constant pressure, the formation enthalpy of a surface defect can be obtained directly from the DFTcalculated energies of the stoichiometric surface (denoted $E_{\text {Ceria }}^{\text {Stoic }}$ ) and either the reduced surface (denoted $E_{\text {Ceria }}^{\text {OxVac }}$ ) or the oxidised surface (denoted $E_{\text {Ceria }}^{\mathrm{CeVac}}$ ). In this way, we may define the formation enthalpy of an oxygen vacancy $\left(\Delta H_{f}^{O x V a c}\right)$ as

$$
\Delta H_{f}^{\text {OxVac }}=E_{\text {Ceria }}^{\text {OxVac }}-\left(E_{\text {Ceria }}^{\text {Stoic }}-\frac{1}{2} \mu_{O_{2}}\right)
$$

where $\mu_{O_{2}}$ is the chemical potential for gas-phase oxygen, taken to be synonymous with the DFT-calculated energy (per molecule) of isolated $\mathrm{O}_{2}$. In similar fashion, the formation enthalpy of a cerium vacancy $\left(\Delta H_{f}^{\mathrm{CeVac}}\right)$ may be defined as

$$
\Delta H_{f}^{\mathrm{CeVac}}=E_{\text {Ceria }}^{\mathrm{CeVac}}-\left(E_{\mathrm{Ceria}}^{\mathrm{Stoic}}+\mu_{\mathrm{O}_{2}}-\mu_{\mathrm{CeO}_{2}}\right)
$$

where $\mu_{\mathrm{CeO}_{2}}$ is the chemical potential for solid ceria, taken to be synonymous with the DFT-calculated energy (per primitive unit cell) of bulk $\mathrm{CeO}_{2}$.

Upon deposition of an adatom, two different forms of adsorption enthalpy may usefully be defined. The first of these describes only the enthalpy change due to the process of adsorption itself, onto whatever surface (stoichiometric, reduced, or oxidised) happens to be involved. That is, we have

$$
\Delta H_{a}^{M / S}=-\left(E_{\mathrm{CeO}_{2}}^{\mathrm{M} / S}-E_{\mathrm{CeO}_{2}}^{S}-E_{M}\right)
$$

in which the symbol $M(=A u / I r)$ indicates the adspecies, and $S=($ Stoic $/ \mathrm{OxVac} / \mathrm{CeVac})$ indicates the stoichiometry of the surface onto which adsorption occurs. Here, $E_{M}$ represents the DFT-calculated energy of a single isolated atom of the relevant adspecies.

The second definition, which applies only to the reduced and oxidised cases, includes a correction to account for the formation enthalpy of the relevant vacancy near which the adatom is adsorbed. In this case, we may write

$$
\Delta \widetilde{H}_{a}^{M / S}=\Delta H_{a}^{M / S}-\Delta H_{f}^{S}
$$

where the tilde distinguishes the symbol used for this second definition from that used for the first. When dealing with a surface under reducing conditions, where oxygen vacancies will be naturally abundant, or under oxidising conditions, where cerium vacancies may be similarly abundant, the $\Delta H_{a}$ definition is arguably the most appropriate to invoke when interpreting the energetics of adsorption. Under redox neutral conditions, in contrast, the $\Delta \widetilde{H}_{a}$ definition is the more suitable, since vacant sites, of either type, are likely to be relatively rare unless induced by the act of adsorption itself.

\section{RESULTS}

\section{A. Stoichiometric and Defective $\mathrm{CeO}_{2}\{111\}$ Surfaces}

The electronic structures of metal oxides are frequently discussed as though they were purely ionic systems, in which the constituent cations and anions acquire integer charges (corresponding to their oxidation states) and covalency plays no role whatsoever. This level of description is, of course, oversimplified and naive, but it is nevertheless fair to say that it does often provide a surprisingly meaningful foundation upon which to build a more accurate and nuanced understanding. In the analyses that follow, therefore, we shall first discuss each system in terms of supposed integer charges, before carefully outlining how the true electronic structure differs from the idealised fiction of this oxidation-state model. 
TABLE I. Mean orbital populations for the stoichiometric $\mathrm{CeO}_{2}\{111\}$ surface (denoted Stoic here and elsewhere). Subscripts (1, 2 or 3 ) indicate that the populations are averaged over the first, second or third trilayer, the latter being central to the slab and hence very nearly bulk-like in nature ( $c f$. the results of our bulk calculation, also tabulated).

\begin{tabular}{lllllllll}
\hline \hline & $\mathrm{O} 2 \mathrm{~s}$ & $\mathrm{O} 2 \mathrm{p}$ & $\mathrm{Ce} 5 \mathrm{~s}$ & $\mathrm{Ce} 5 \mathrm{p}$ & $\mathrm{Ce} 6 \mathrm{~s}$ & Ce 5d & Ce 4f & Ce 6p \\
\hline Stoic $_{1}$ & 1.88 & 4.79 & 1.98 & 5.91 & 0.25 & 1.47 & 0.90 & 0.15 \\
Stoic $_{2}$ & 1.87 & 4.79 & 1.98 & 5.90 & 0.24 & 1.49 & 0.89 & 0.19 \\
Stoic $_{3}$ & 1.87 & 4.78 & 1.98 & 5.89 & 0.25 & 1.49 & 0.89 & 0.19 \\
\hline Bulk & 1.87 & 4.78 & 1.98 & 5.89 & 0.20 & 1.52 & 0.90 & 0.23 \\
\hline \hline
\end{tabular}

In the case of bulk ceria, for example, we have already mentioned that the cerium cations adopt an oxidation number of +4 , while the oxygen anions adopt an oxidation number of -2 , but the actual charges that can reasonably be attributed to these species are of much smaller magnitude. Various approaches can be taken to apportion charge between different components within a system, each giving subtly different results and having their own unique advantages and disadvantages, but if we were to make use of the commonly applied Mulliken methodology, ${ }^{47}$ we would conclude that the cerium cations acquire a charge of only $+1.30|e|$ and the oxygen anions a counterbalancing charge of $-0.65|e|$. In the oxidationstate model, the Ce $6 s, 5 d$ and $4 f$ states would be entirely depopulated in order to supply the electrons necessary for the complete occupancy of the O $2 p$ states. In the more nuanced picture revealed by the Mulliken analysis, the $\mathrm{Ce} 6 s$ states are indeed substantially (albeit not entirely) depopulated, but the $4 f$ states retain very nearly one electron per ion (as they would in the neutral atom) and the $5 d$ states actually hold about one and a half electrons per ion (which is more than would be expected for the neutral atom). Furthermore, some of the electrons lost from these states (about one fifth of an electron per ion) are actually redistributed into Ce $6 p$ states, which one would expect to be empty in an isolated cerium atom. Correspondingly, the $\mathrm{O} 2 p$ states need to accommodate only around four and three quarter electrons per ion, rather than the six that would be expected in the oxidation-state model, while the $\mathrm{O}$ $2 s$ states hold slightly fewer than two electrons per ion. Indeed, the distribution in energy of the occupied fractions of the Ce $6 s, 5 d, 4 f$ and $6 p$ states matches precisely that of the $\mathrm{O} 2 p$ states, suggesting strongly that a substantial degree of covalency is involved in stabilisation of the bulk material, contrary to the purely ionic picture implied by the nominal oxidation states.

\section{Stoichiometric $\mathrm{CeO}_{2}\{111\}$}

Turning now to the stoichiometric $\mathrm{CeO}_{2}\{111\}$ surface, the oxidation-state model would predict the same oxidation numbers of +4 and -2 for the cations and anions throughout the slab employed in our calculations, and we might reasonably expect the true electronic structure to be similarly insensitive to the presence of a surface. To investigate, let us consider in detail the slab's PDOS, presented in Fig. 2. The O $2 s$ electrons and the Ce $5 s$ and $5 p$ electrons lie substantially more than $8 \mathrm{eV}$ below the Fermi level (which we take to be syn-

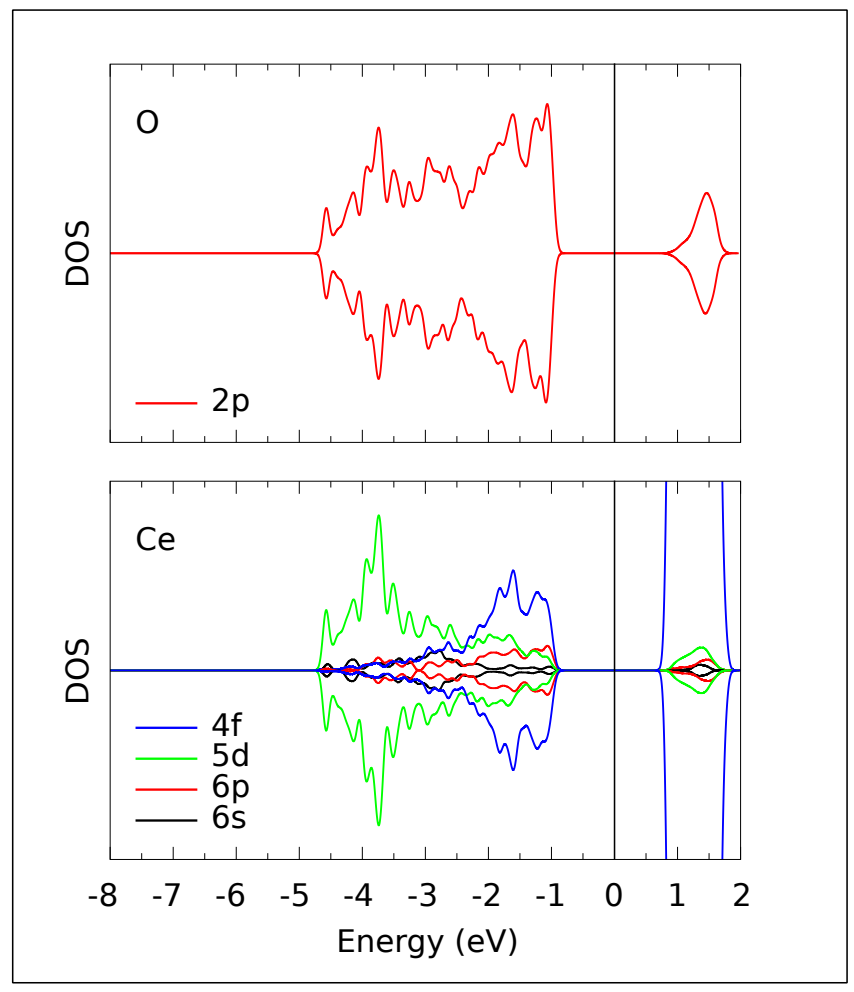

FIG. 2. PDOS for the stoichiometric $\mathrm{CeO}_{2}\{111\}$ surface. Traces in the upper and lower half of each panel are for the two spin channels, the Fermi level is indicated at $0.0 \mathrm{eV}$. The arbitrary vertical scale is common to all traces except for the $\mathrm{O} 2 p$ states, which have been scaled down by a factor of four relative to the others.

onymous with the electronic chemical potential) and are thus not depicted. The $\mathrm{O} 2 p$ levels are (as in the bulk) not quite fully occupied, having sufficient weight below the Fermi level to accommodate only about four and three quarter electrons per oxygen ion. Of these, around one eighth of an electron per ion is to be found in states lying within a minor band situated more than $14 \mathrm{eV}$ below the Fermi level (not shown in the figure) with the remainder distributed across a major band spanning the range $0.75-4.75 \mathrm{eV}$ below the Fermi level. We find occupied Ce $5 d$ states throughout the exact same energy ranges, of which around one fifth of an electron per cerium ion is energetically matched with the $\mathrm{O} 2 p$ minor band, while around one and a quarter electrons per cerium ion are energetically matched with the major. Similarly, the Ce $4 f$ states are also not depopulated, and sufficient weight to accommodate 
TABLE II. Changes in selectively summed orbital populations upon the creation of an oxygen vacancy ( OxVac) or a cerium vacancy $(\mathrm{CeVac})$ at an otherwise stoichiometric $\mathrm{CeO}_{2}\{111\}$ surface (whose orbital populations are tabulated in Table I). Subscripts (1, 2 or 3 ) indicate that the populations are summed over the first, second or third trilayer. In each trilayer, summations are carried out over a single $(2 \times 2)$ surface unit cell.

\begin{tabular}{|c|c|c|c|c|c|c|c|c|}
\hline & $\Delta(\mathrm{O} 2 \mathrm{~s})$ & $\Delta(\mathrm{O} 2 \mathrm{p})$ & $\Delta(\mathrm{Ce} 5 \mathrm{~s})$ & $\Delta(\mathrm{Ce} 5 \mathrm{p})$ & $\Delta(\mathrm{Ce} 6 \mathrm{~s})$ & $\Delta(\mathrm{Ce} 5 \mathrm{~d})$ & $\Delta(\mathrm{Ce} 4 \mathrm{f})$ & $\Delta(\mathrm{Ce} 6 \mathrm{p})$ \\
\hline $\mathrm{OxVac}_{1}$ & -1.91 & -4.49 & 0.06 & 0.17 & -0.07 & -0.15 & 0.52 & -0.12 \\
\hline $\mathrm{OxVac}_{2}$ & 0.00 & 0.02 & 0.01 & 0.00 & 0.00 & -0.03 & 0.01 & -0.01 \\
\hline $\mathrm{OxVac}_{3}$ & 0.01 & 0.00 & 0.00 & 0.00 & 0.01 & -0.01 & -0.01 & 0.01 \\
\hline $\mathrm{CeVac}_{1}$ & 0.20 & -1.31 & -1.98 & $\begin{array}{l}-5.83 \\
\end{array}$ & -0.35 & -1.34 & -0.94 & -0.17 \\
\hline $\mathrm{CeVac}_{2}$ & 0.02 & -0.07 & 0.00 & -0.02 & 0.02 & -0.02 & 0.01 & -0.04 \\
\hline $\mathrm{CeVac}_{3}$ & 0.00 & 0.00 & 0.00 & -0.01 & 0.00 & 0.00 & -0.01 & -0.01 \\
\hline
\end{tabular}

about nine tenths of an electron per cerium ion is distributed throughout the same energy range as the major band of occupied $\mathrm{O} 2 p$ states. That said, the vast majority of the $\mathrm{Ce}$ $4 f$ states do remain fully localised, lying (unoccupied) in a band spanning the range $0.75-1.75 \mathrm{eV}$ above the Fermi level. Some very slight occupancy of Ce $6 s$ and $6 p$ states is also apparent, but amounting to no more than about a quarter of an electron and a fifth of an electron per cerium ion respectively, both associated with states spanning the same energy range as the major band of $\mathrm{O} 2 p$ states. Mulliken analysis indicates charges in the ballpark of $+1.32|e|$ for cerium and $-0.66|e|$ for oxygen, with very little variation between those ions located close to the slab surfaces and those located close to the slab centre. Mean orbital populations averaged separately for each of the top three trilayers are presented in Table I for ease of reference. In short, the electronic structure of our slab is highly consistent with that of the bulk material, differing only very slightly in its quantitative detail but not at all in its qualitative features.

\section{Reduced $\mathrm{CeO}_{2}\{111\}$}

Unsurprisingly, the creation of an oxygen vacancy at the surface (calculated formation enthalpy $2.32 \mathrm{eV} ; c f 2.23 \mathrm{eV}$ given by Zhang et $\mathrm{al}^{11}$ ) induces much more significant modification of the calculated electronic structure. Here, the oxidation-state model would predict that abstraction of a neutral oxygen atom from the surface must leave behind the two electrons that were previously associated with its presence in the surface as an oxygen anion. These two electrons would then be most readily accommodated by the surface in $\mathrm{Ce}$ $4 f$ states localised on two out of the three cerium cations surrounding the vacancy itself. ${ }^{13}$ These two cerium cations would thus adopt an oxidation number of +3 , and may sensibly be described as reduced. We might justifiably question, however, whether this neat scenario survives contact with the more realistic situation, described above, in which both cations and anions carry non-integer charges much smaller than those implied by a naive acceptance of the oxidation-state model. In fact, the answer is resoundingly in the affirmative, albeit with important caveats. Examination of the PDOS for a slab incorporating an oxygen vacancy (Fig. 3) clearly reveals the existence of two very nearly degenerate majority-polarised

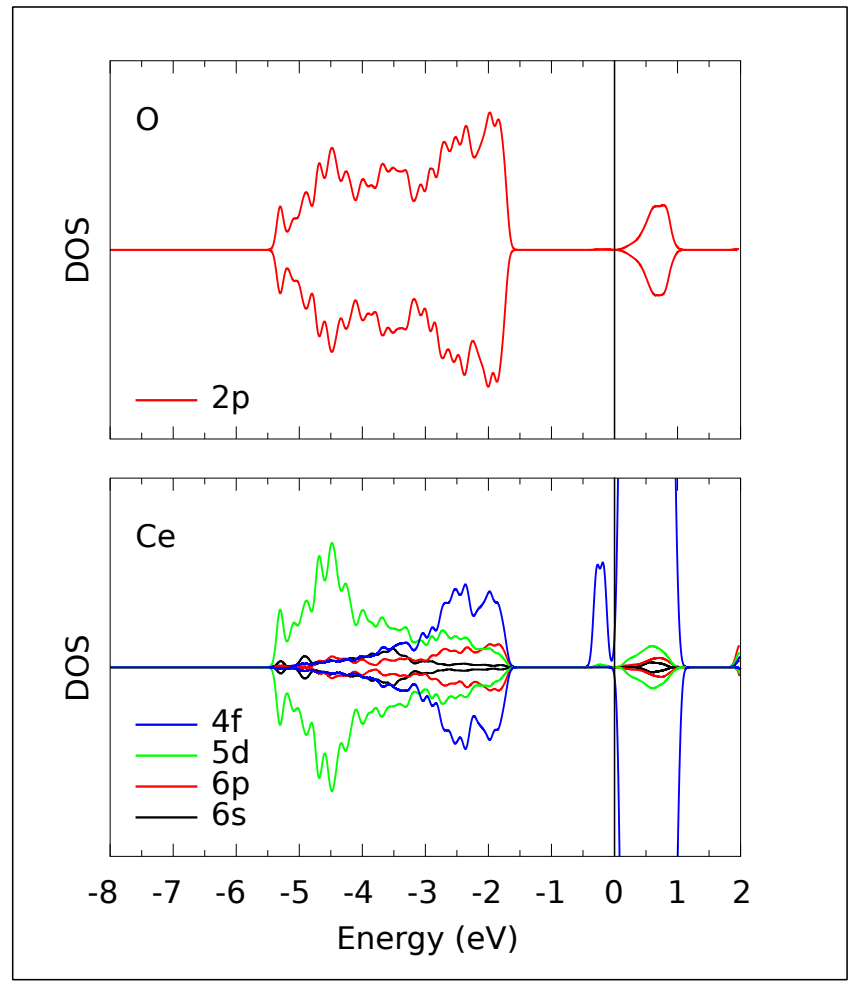

FIG. 3. PDOS for a $\mathrm{CeO}_{2}\{111\}$ surface modified with a single oxygen vacancy. Details of the presentation are as per Fig. 2 .

peaks in the Ce $4 f$ channel, positioned just below the Fermi level, each accommodating one electron as the oxidation-state model would predict.

Such an observation leads to a seeming paradox, however, given that the departure of a neutral oxygen atom requires the surface to accommodate far fewer "leftover" electrons than the oxidation-state model would have suggested. It is important to note, therefore, that the total occupancy of the Ce $4 f$ channel actually increases by only around two fifths of an electron relative to the stoichiometric surface, since the states making up the two new Ce $4 f$ peaks are drawn largely from those originally lying beneath the Fermi level, as opposed to those originally lying above it. Modulation of orbital population amongst cerium ions is almost entirely confined to the uppermost trilayer (Table II) and especially to just two such 
ions. These are confirmed by Mulliken analysis to attain a net charge of $+1.22|e|$, which is just a little less than the charge of $+1.30|e|$ found on all the others. This is consistent with the notion that they have been reduced, but again the decrease in net charge is of much lower magnitude than the change in oxidation number from +4 to +3 might suggest.

Whilst the presence of majority-polarised occupied states just below the main (unoccupied) Ce $4 f$ band matches exactly the predictions of the oxidation-state model, then, their origin requires a much more subtle explanation. Specifically, it is worth noting that the overall increase in Mulliken population of the Ce $4 f$ states on the reduced ions (just over a quarter of an electron per ion) is largely compensated by a concomitant drop in Mulliken population of the Ce $5 d$ states on those same ions (a loss of almost one sixth of an electron per ion). The most striking signature of change in the $\mathrm{Ce} 4 f$ states is therefore not the effect they have on the overall charge of the reduced ions, but rather in their spin state, which switches from essentially spin-neutral to a net spin of $0.98 \mu_{B}$ per ion in our calculations. Almost the whole of this net spin (c.95\%) is associated directly with the Ce $4 f$ states, as can be seen not only from the Mulliken analysis but also by visualising the net spin density of the system. In Fig. 4.a, for example, we not only see regions of high local net spin density associated with the two reduced cerium ions, but also observe a distribution of this spin that is consistent with spin-asymmetry in the occupation of a single Ce $4 f$ orbital located on each ion.

Notwithstanding these details, the mere existence of majority-polarised occupied $\mathrm{Ce} 4 f$ peaks does give rise to one very important feature of the vacancy's electronic structure, namely the pinning of the Fermi level at the upper margin of the original band gap. In consequence, the main valence complex of ceria, which had occupied the binding-energy range $0.75-4.75 \mathrm{eV}$ in the case of the stoichiometric surface, is down-shifted (relative to the Fermi level) by around $0.75 \mathrm{eV}$ in the presence of the vacancy. In the case of an isolated vacancy embedded in an expanse of otherwise stoichiometric surface, this would imply very significant band bending in the locality of the vacancy, with consequences for electronic transport properties in the vicinity. The behaviour of defects in wide band-gap semiconductors provides a useful point of reference in this regard. It should be noted, however, that this aspect of the electronic structure is amongst those most likely to be sensitive to the precise value employed for the $U$ parameter.

\section{Oxidised $\mathrm{CeO}_{2}\{111\}$}

In contrast to the electronic structure of the reduced surface, that of an oxidised surface, modified by the creation of a cerium vacancy (calculated formation enthalpy $5.21 \mathrm{eV} ; c f$ $4.67 \mathrm{eV}$ given by Zhang $\left.e t a l^{11}\right)$ is dominated by the emergence of distinct unoccupied peaks within the band gap, rather than occupied ones. Within the oxidation-state model, abstraction of a neutral cerium atom from the surface requires the removal of four electrons, and these might be expected to be drawn from the oxygen anions adjacent to the vacancy site. The expectation, therefore, is that these four anions would be
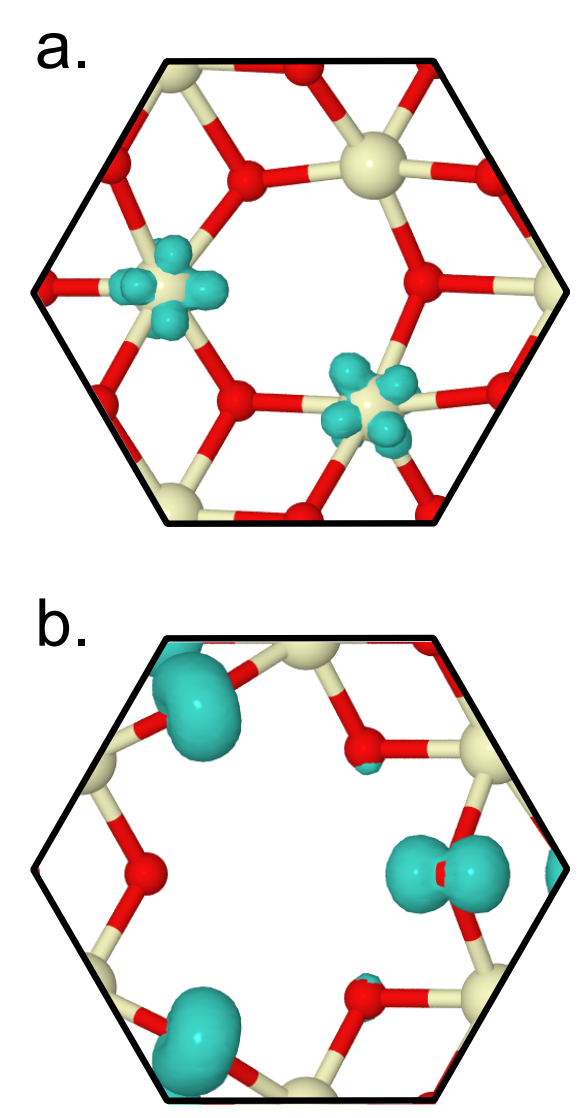

FIG. 4. Orthographic top-down views of (a) an oxygen vacancy, and (b) a cerium vacancy. Only top-layer atoms are shown, for reasons of clarity, with cerium depicted by grey spheres and oxygen by red ones. The region shown in each case is a Voronoi cell of area equal to the $(2 \times 2)$ cell used in the calculations. Isosurfaces indicate regions in which the magnitude of the net spin density exceeds $0.1 \mu_{B} . \AA^{-3}$ (where $\mu_{B}$ is the Bohr magneton). In these cases, all such regions are locally dominated by the overall majority spin species.

oxidised, from an oxidation number of -2 to an oxidation number of -1 . Once again, the question is to what extent this simple view is borne out by the electronic structure revealed in the PDOS from our slab calculations (Fig. 5) and once again the answer is cautiously positive. Specifically, a sequence of four unoccupied minority-polarised peaks is visible in the $\mathrm{O}$ $2 p$ channel, just above the Fermi level, but these are clearly associated with a similar sequence of peaks in the Ce $4 f$ channel, indicating that they are representative of covalent mixed states and not wholly localised on just the anions. With both types of orbital contribution included, however, each peak is capable of accommodating precisely one electron, as expected from the oxidation-state model. Nevertheless, the total occupancy of the $\mathrm{O} 2 p$ channel is decreased by only about one and a third electrons, with at least half of the weight contributing to the four new peaks being drawn from states that lay above the Fermi level at the parent stoichiometric surface. The effect is almost entirely confined to the uppermost trilayer (Table II) with only very minor changes notable in the second layer and 
below.

Indeed, looking more closely at individual ions, our Mulliken analysis reveals that it is the three oxygen ions in the upper portion of the trilayer lying closest to (and very nearly equidistant from) the vacancy that exhibit the most significant drop in $\mathrm{O} 2 p$ population, two losing just over a third of an electron each, and the other losing just under. This implies a spontaneous reduction in the system's electronic symmetry, concomitant with a modest symmetry-breaking distortion of the geometry (i.e. a Jahn-Teller distortion). The other oxygen ion in the upper portion of the trilayer, lying furthest from the vacancy within our simulation cell, exhibits virtually no change in $2 p$ population. In contrast, amongst the four oxygen ions belonging to the lower portion of the trilayer, the three lying closest to the vacancy are minimally altered, while the one lying furthest from the vacancy loses almost a fifth of an electron from its $\mathrm{O} 2 p$ states.

Spontaneous lowering of symmetry (noted above in relation to charge and geometry) is also reflected in the spatial distribution of spin, as depicted visually in Fig. 4.b. The two oxygen ions located towards the left of the panel each acquire net spins of $1.21 \mu_{B}$ (these are the two whose $2 p$ states are most depleted upon vacancy formation) while the oxygen ion located towards the right of the panel acquires a net spin of only $0.93 \mu_{B}$ (the only other ion in the upper portion of the trilayer whose $\mathrm{O} 2 p$ population is significantly altered). Just one oxygen ion from the lower portion of the trilayer acquires any appreciable net spin, amounting to $0.72 \mu_{B}$, and unsurprisingly this is the one lying furthest from the vacancy itself (consistent with the pattern of charge variation described above). In all cases, the net spin of these ions arises predominantly (90-98\%) from the O $2 p$ states, with only a very minor contribution from $\mathrm{O} 2 s$. By way of contrast, the net spin acquired by cerium ions is rather small, averaging just $0.13 \mu_{B}$ per ion within the upper trilayer (counterpolarised with respect to the net spin of the oxygen ions) and entirely negligible in deeper trilayers.

Akin to the case of the oxygen vacancy, perhaps the main consequence of the spin-polarised peaks in the band gap (regardless of their origin) is that they pin the Fermi level. This time, however, they pin it at the lower margin of the original band gap, rather than the upper, and the main valence complex of ceria is consequently up-shifted relative to the Fermi level by around $0.5 \mathrm{eV}$ relative to the stoichiometric case. As noted above for the reduced surface, pinning of the Fermi level would likely give rise to significant band bending in the vicinity of an isolated defect surrounded by an expanse of otherwise stoichiometric surface, albeit now in the opposite sense.

\section{B. Adsorption of Gold on $\mathrm{CeO}_{2}\{111\}$ Surfaces}

As highlighted in the introduction to this work, adsorption of gold on ceria has been studied extensively, and much of the foundational work on this topic is summarised in the review by Zhang et al. ${ }^{13}$ Although such issues as the preferred adsorption sites have already been settled, not only on stoichiometric but also on defective surfaces, we have nevertheless

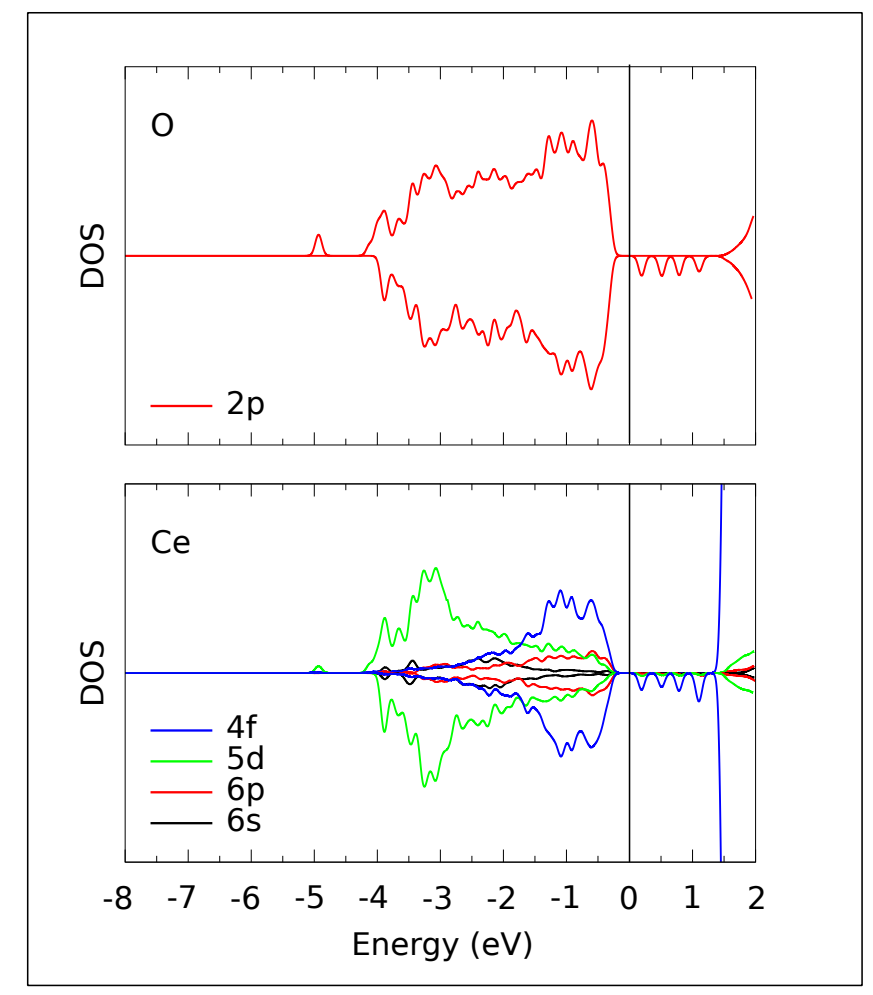

FIG. 5. PDOS for a $\mathrm{CeO}_{2}\{111\}$ surface modified with a single cerium vacancy. Details of the presentation are as per Fig. 2.

confirmed these afresh in the present work (Fig. 6). We determine that a single gold adatom adsorbs preferentially into a site bridging between two top-layer oxygen ions on the stoichiometric surface, with a $\Delta H_{a}$ binding enthalpy (calculated as per Eqn. 3) of $1.16 \mathrm{eV}$, comparing very well with the adsorption heat of $1.17 \mathrm{eV}$ previously calculated by Zhang et al. ${ }^{11}$ On a surface modified by the presence of either an oxygen or a cerium vacancy, however, such an adatom adsorbs preferentially into the vacant site itself, with a $\Delta H_{a}$ binding enthalpy of $2.36 \mathrm{eV}$ or $6.15 \mathrm{eV}$ respectively, relative to the relevant clean defective surface. These findings again accord reasonably well with prior results, where adsorption heats of $2.75 \mathrm{eV}$ and $5.94 \mathrm{eV}$ have previously been calculated. ${ }^{8}$ Including the respective formation enthalpies of oxygen and cerium vacancies our $\Delta \widetilde{H}_{a}$ binding enthalpies (calculated as per Eqn. 4) correspond to $0.04 \mathrm{eV}$ and $0.94 \mathrm{eV}$, relative to the stoichiometric surface.

In light of these findings, it is worthwhile considering implications for the stability of single-atom gold on the ceria surface. We calculate the bulk cohesive energy of gold to be $3.08 \mathrm{eV}$ per atom (cf. $3.81 \mathrm{eV}$ by experiment) which is considerably greater than either of the $\Delta \widetilde{H}_{a}$ binding enthalpies. That is to say, there is no strong driving force, under redox-neutral conditions, toward displacement of either oxygen or cerium from the surface in order to accommodate gold adatoms at vacancy sites, as opposed to their forming large bulk-like gold clusters. Adsorption of single gold adatoms onto the stoichiometric surface will be similarly disfavoured relative to cluster formation. Only when oxygen-rich conditions would natu- 
TABLE III. Changes in selectively summed orbital populations upon adsorption of species $M(=A u / I r)$ onto different $\mathrm{CeO}_{2}\{111\}$ surfaces (Stoic indicates adsorption on the stoichiometric surface; OxVac indicates adsorption on the reduced (oxygen vacancy) surface; and CeVac indicates adsorption on the oxidised (cerium vacancy) surface). These are changes additional to any changes due to formation of vacancies (tabulated in II). Subscripts (1, 2 or 3) indicate that the populations are summed over the first, second or third trilayer. In each trilayer, summations are carried out over a single $(2 \times 2)$ surface unit cell. Changes in adatom orbital populations are stated relative to the nominal electronic configuration of an isolated atom $\left(6 \mathrm{~s}^{1} 5 \mathrm{~d}^{10}\right.$ for $\mathrm{Au}$, and $6 \mathrm{~s}^{2} 5 \mathrm{~d}^{7}$ for Ir).

\begin{tabular}{|c|c|c|c|c|c|c|c|c|c|c|}
\hline & $\Delta(\mathrm{O} 2 \mathrm{~s})$ & $\Delta(\mathrm{O} 2 \mathrm{p})$ & $\Delta(\mathrm{Ce} 5 \mathrm{~s})$ & $\Delta($ Ce $5 p)$ & $\Delta(\mathrm{Ce} 6 \mathrm{~s})$ & $\Delta(\operatorname{Ce} 5 \mathrm{~d})$ & $\Delta(\mathrm{Ce} 4 \mathrm{f})$ & $\Delta($ Ce 6p) & $\Delta(\mathrm{M} \mathrm{6s})$ & $\Delta(\mathrm{M} \mathrm{5d})$ \\
\hline$\overline{A u / \text { Stoic }_{1}}$ & 0.00 & 0.23 & 0.03 & 0.11 & 0.04 & -0.10 & 0.26 & 0.01 & -0.18 & -0.37 \\
\hline $\mathrm{Au} /$ Stoic $_{2}$ & 0.00 & -0.01 & 0.00 & -0.01 & 0.01 & -0.01 & 0.00 & 0.00 & - & - \\
\hline $\mathrm{Au} / \mathrm{Stoic}_{3}$ & 0.01 & 0.00 & 0.00 & 0.00 & 0.01 & -0.01 & -0.01 & 0.01 & - & - \\
\hline $\mathrm{Au} / \mathrm{OxVac}_{1}$ & 0.00 & -0.18 & -0.03 & -0.07 & 0.19 & 0.22 & -0.39 & 0.04 & 0.54 & -0.22 \\
\hline $\mathrm{Au} / \mathrm{OxVac}_{2}$ & 0.00 & -0.01 & -0.01 & -0.01 & 0.01 & 0.01 & -0.01 & -0.04 & - & - \\
\hline $\mathrm{Au} / \mathrm{OxVac}_{3}$ & 0.00 & -0.01 & 0.00 & 0.00 & 0.00 & 0.01 & 0.00 & 0.00 & - & - \\
\hline $\mathrm{Au} / \mathrm{CeVac}_{1}$ & -0.08 & 1.47 & 0.03 & 0.11 & 0.09 & -0.43 & 0.20 & 0.02 & -0.28 & -1.23 \\
\hline $\mathrm{Au} / \mathrm{CeVac}_{2}$ & -0.02 & 0.06 & 0.00 & 0.00 & -0.02 & 0.01 & -0.02 & 0.07 & - & - \\
\hline $\mathrm{Au} / \mathrm{CeVac}_{3}$ & 0.01 & 0.00 & 0.00 & 0.01 & 0.00 & 0.00 & 0.00 & 0.01 & - & - \\
\hline Ir $/$ Stoic $_{1}$ & -0.04 & 0.43 & 0.10 & 0.32 & -0.12 & -0.49 & 0.81 & -0.05 & -1.23 & 0.21 \\
\hline Ir/Stoic 2 & 0.00 & 0.02 & 0.00 & 0.02 & 0.01 & -0.02 & 0.01 & -0.03 & - & - \\
\hline Ir $/$ Stoic $_{3}$ & 0.00 & 0.00 & 0.00 & 0.00 & -0.01 & 0.02 & -0.01 & 0.00 & - & - \\
\hline $\mathrm{Ir} / \mathrm{OxVac}_{1}$ & 0.02 & 0.09 & 0.03 & 0.08 & -0.01 & -0.18 & 0.26 & 0.06 & -0.94 & 0.41 \\
\hline $\mathrm{Ir} / \mathrm{OxVac}_{2}$ & 0.01 & 0.13 & 0.02 & 0.09 & -0.03 & -0.15 & 0.19 & -0.06 & - & - \\
\hline $\mathrm{Ir} / \mathrm{OxVac}_{3}$ & -0.01 & 0.01 & 0.00 & -0.01 & -0.01 & 0.02 & 0.00 & -0.02 & - & - \\
\hline $\mathrm{Ir} / \mathrm{CeVac} c_{1}$ & -0.17 & 1.46 & 0.03 & 0.08 & 0.05 & -0.34 & 0.29 & 0.00 & -1.47 & -0.27 \\
\hline $\mathrm{Ir} / \mathrm{CeVac}_{2}$ & -0.01 & 0.23 & 0.02 & 0.09 & -0.05 & -0.09 & 0.10 & 0.02 & - & - \\
\hline $\mathrm{Ir} / \mathrm{CeVac}_{3}$ & 0.00 & 0.01 & 0.00 & 0.01 & 0.00 & 0.01 & 0.00 & 0.01 & - & - \\
\hline
\end{tabular}

rally favour the reduced surface, or when oxygen-poor conditions would naturally favour the oxidised surface, will adsorption of single gold adatoms into pre-existing oxygen or cerium vacancies (as appropriate) be favourable over adsorption into stoichiometric sites. Even then, however, only for adsorption into the cerium will the $\Delta H_{a}$ binding enthalpy exceed the cohesive energy of bulk gold. The presence of single-atom gold at the oxygen vacancy sites of reduced ceria cannot, therefore, be attributed to thermodynamic stability. Instead, we note that migration of a gold adatom from such a site to a neighbouring patch of stoichiometric surface will involve surmounting a barrier of at least $1.20 \mathrm{eV}$ (i.e. the difference between $\Delta H_{a}$ binding enthalpies of $2.36 \mathrm{eV}$ at the vacancy and $1.16 \mathrm{eV}$ on the stoichiometric surface) and hence may be kinetically hindered at low-to-moderate temperatures, preventing sintering and/or ripening under these conditions. Single-atom gold in cerium vacancy sites would, on the other hand, appear to be thermodynamically stable at high temperatures, albeit only under sufficiently oxidising conditions. That is, so long as cerium vacancies are plentiful, gold adatoms ought to singly occupy such sites in preference to forming clusters, regardless of the system's ability to overcome any kinetic barriers to sintering and/or ripening that may exist.

\section{Au on Stoichiometric $\mathrm{CeO}_{2}\{111\}$}

Focussing now upon the electronic structures associated with single-atom gold adsorption, we may once again benefit by comparing the PDOS in each case against the predictions of the oxidation-state model. On the stoichiometric surface, for example, the fate of the Au $6 s$ states might be expected to play a role of central importance. In the isolated neutral atom, these states are, of course, singly occupied and might be considered both as a likely destination for electrons donated to the atom if it were to become anionic, and also as a possible source for electrons donated by the atom if it were to become cationic. The high electronegativity of gold would normally argue against the latter view, but in the present circumstance it is worth noting that the stoichiometric surface may also be considered highly electronegative, possessing a very substantial density of unoccupied states in the vicinity of the Fermi level.

Examination of the PDOS for the preferred adsorption geometry (Fig. 7) confirms that a sharp majority-polarised peak does indeed arise below the lower edge of the main Ce $4 f$ band, dropping beneath the Fermi level and accommodating precisely one electron, as noted in several prior works. ${ }^{6,8-10,15}$ This charge has previously been explained as originating from depopulation of the Au $6 s$ state, ${ }^{6}$ but we note here that although one finds an unoccupied peak of Au $6 s$ character at around $1.6 \mathrm{eV}$ above the Fermi level, its weight (including both spin components) is sufficient to account for only a little more than a single electron, not two. Moreover, occupied states of $\mathrm{Au} 6 \mathrm{~s}$ character are distributed throughout the energy range associated with the main valence complex (somewhat weighted towards the extremes of that range) and retain charge amounting to at least four fifths of an electron. The main electron donors to the majority-polarised $\mathrm{Ce} 4 f$ state seem, therefore, to be the Au $5 d$ states, whose occupancy drops by about a third of an electron compared with the isolated atom, and the other occupied Ce $4 f$ states, whose occupancy drops 

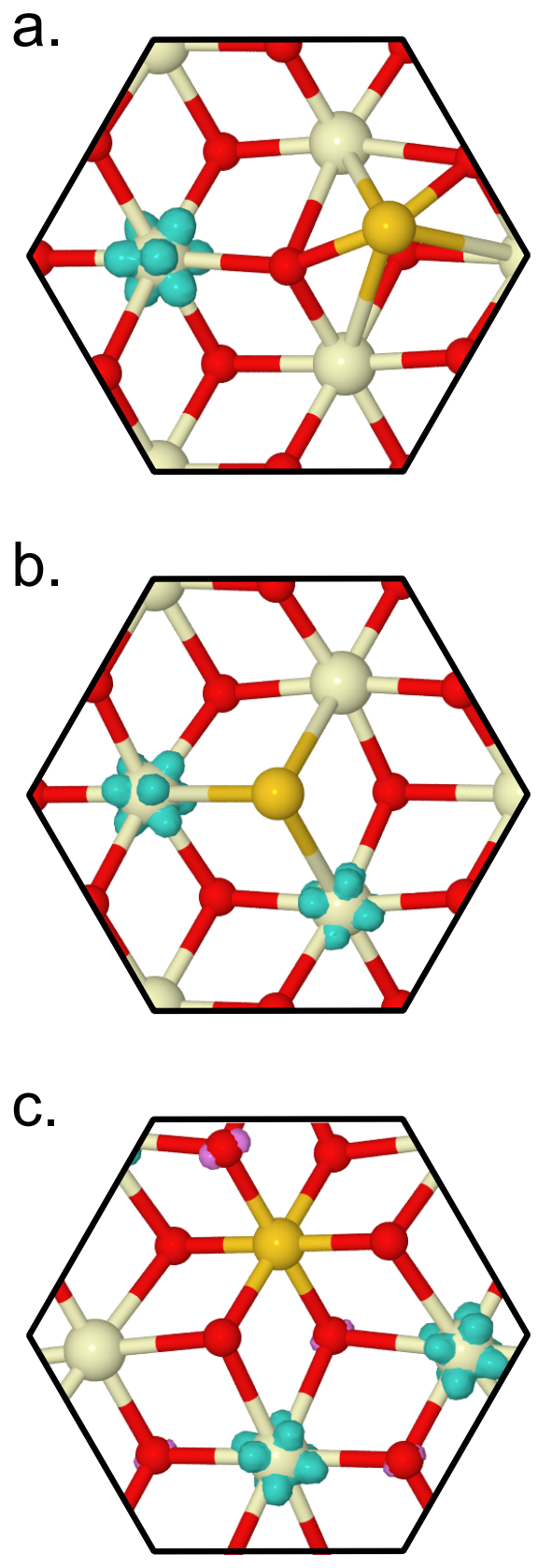

FIG. 6. Orthographic top-down views of a gold atom adsorbed (a) on the stoichiometric surface, (b) at an oxygen vacancy, and (c) at a cerium vacancy. Details of the presentation are as per Fig. 4. Isosurfaces indicate regions in which the magnitude of the net spin density exceeds $0.1 \mu_{B} . \AA^{-3}$, with turquoise indicating that the overall majority spin species is locally dominant, and lilac indicating that the overall minority spin species is locally dominant.

by two thirds of an electron compared with the stoichiometric surface. Mulliken analysis (Table III) confirms that the adatom acquires a net charge of $+0.55|e|$, consistent with the observed depletion of the Au $5 d$ states alongside the retention of almost an entire electron in the $\mathrm{Au} 6 s$ states, albeit the latter are now heavily involved in covalent interactions with $\mathrm{O}$

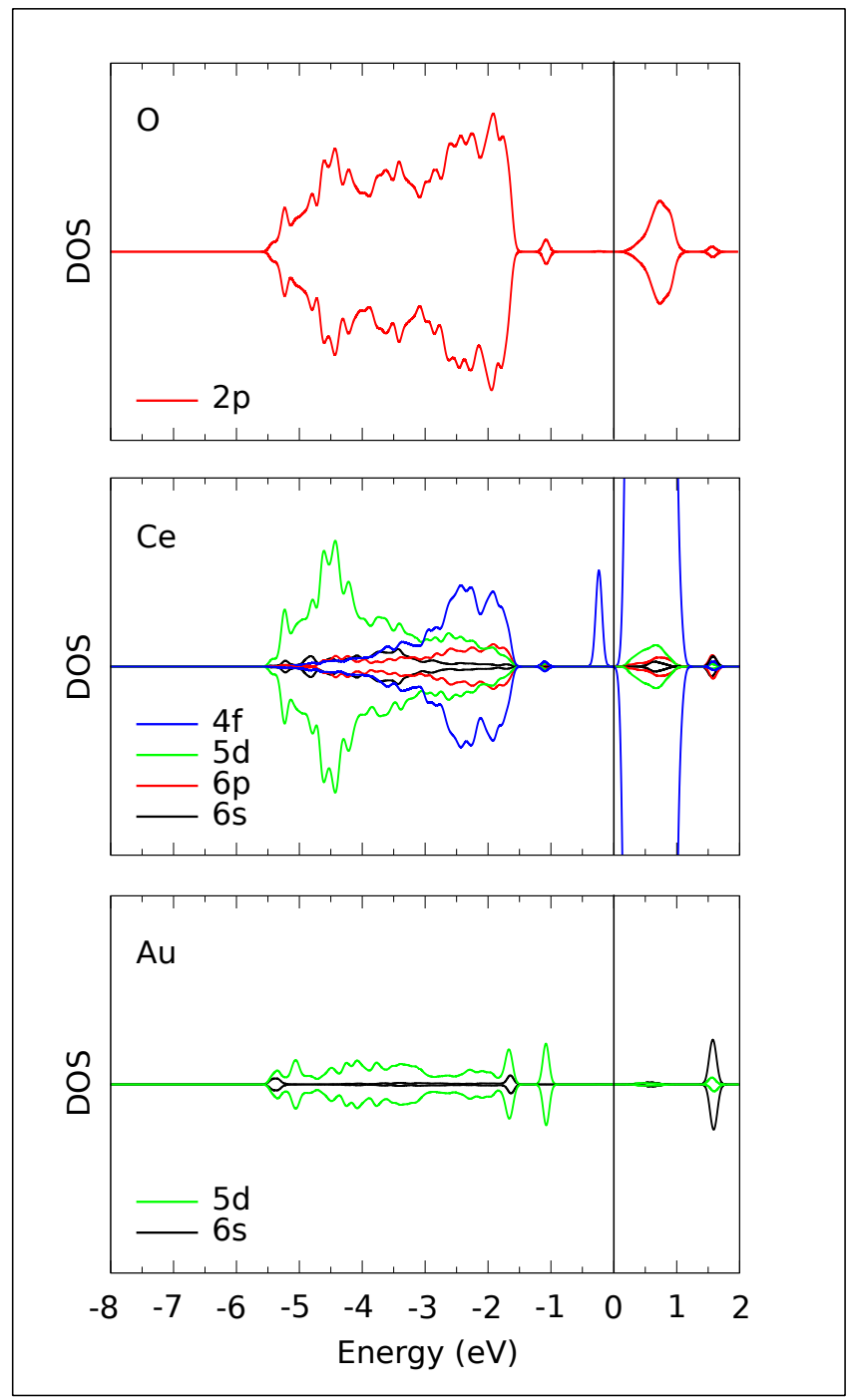

FIG. 7. PDOS for a single Au adatom adsorbed onto a stoichiometric $\mathrm{CeO}_{2}\{111\}$ surface. Details of the presentation are as per Fig. 2.

$2 p$ states. Adsorbed gold does, therefore, achieve a distinctive cationic state on the stoichiometric surface, but not quite in the manner predicted by the oxidation-state model and not to anything like the degree that may naively have been anticipated.

Addressing the spatial localisation of spin, this turns out to be associated entirely with the single cerium atom in the uppermost trilayer with which the adatom does not covalently interact. Whilst the change in overall charge for this ion is rather negligible (the others in the trilayer are slightly more perturbed) it nevertheless acquires a net spin of $1.01 \mu_{B}$, consistent with the existence of a single unpaired electron. Unsurprisingly, this spin asymmetry is predominantly (c.98\%) associated with Ce $4 f$ states, and this may not only be confirmed via Mulliken analysis, but also directly inferred by visualisation of the net spin density (Fig. 6.a). Note that the depletion of electrons from the gold adatom is not accompanied by any accumulation of net spin in that vicinity, which 
is entirely consistent with the observation that $\mathrm{Au} 5 d$ and $6 s$ states remain well away from the Fermi level upon adsorption (Fig. 7). Nevertheless, a small peak is detached from the main $4 d$ band of $\mathrm{Au}$, lying not much more than $1 \mathrm{eV}$ below the Fermi level, and possibly accounting to the enhanced binding of molecular species reported previously for a single gold adatom on stoichiometric ceria. ${ }^{6}$

\section{Au on Reduced $\mathrm{CeO}_{2}\{111\}$}

Regarding adsorption of gold into an oxygen vacancy, it will be recalled that the electronic structure of the empty vacancy was dominated by the existence of two singly occupied Ce $4 f$ states just below the Fermi level. Given the significant electronegativity of gold, the oxidation-state model of binding predicts that one of these would donate its electron to the Au $6 s$ state, inducing the adatom to adopt its anionic configuration. As ever, the situation revealed by examination of the PDOS (Fig. 8) bears a passing resemblance to this scenario, but with important points of difference. Here, we do indeed observe that one of the two singly occupied Ce $4 f$ states associated with the empty vacancy (Fig. 3) shifts from just below the Fermi level to just above, liberating an electron that must be accommodated elsewhere in the electronic structure. Moreover, a distinct peak of Au $6 s$ character may now be observed at $1.3 \mathrm{eV}$ below the Fermi level, but its weight is sufficient (including both spin components) to accommodate only a single electron, not the two that would be consistent with the adatom's suggested anionic condition. Additional states of Au $6 s$ character are distributed throughout the energy range of the main valence complex of ceria, accommodating a further half electron, but this is partially offset by a decrease in the occupied fraction of $\mathrm{Au} 5 d$ states equivalent to around one fifth of an electron compared with the isolated atom.

All of this is consistent with our Mulliken analysis (Table III) which indicates a net charge of just $-0.32|e|$ associated with the gold adatom in the vacancy site, but begs the question of where the remaining electron from the depopulated $\mathrm{Ce} 4 f$ state has been accommodated. The answer turns out to be that the occupancy of other Ce $4 f$ states increases by around three fifths of an electron compared with the situation pertaining to the empty vacancy, while that of the Ce $5 d$ states increases by around a quarter of an electron. Binding of the adatom to the surface is achieved, therefore, not primarily by ionic means, but largely by the strengthening of covalent interactions between $\mathrm{O} 2 p$ states and the Ce $5 d$ and $4 f$ states.

One further important point remains to be made, however, concerning the spatial localisation of spin associated with the majority-polarised $\mathrm{Ce} 4 f$ peaks either side of the Fermi level. Although the value of $U=3 \mathrm{eV}$ used in our calculations successfully ensures an insulating solution, with only a single sharp peak in the spectrum falling below the Fermi level, the state corresponding to that peak does not immediately appear to be fully localised on just one of the top-layer cerium atoms. Instead, we note that two of the neighbouring cerium ions acquire net majority-spin moments of $0.52 \mu_{B}$ and $0.61 \mu_{B}$, as can readily be reconciled with our visualisation of the net spin

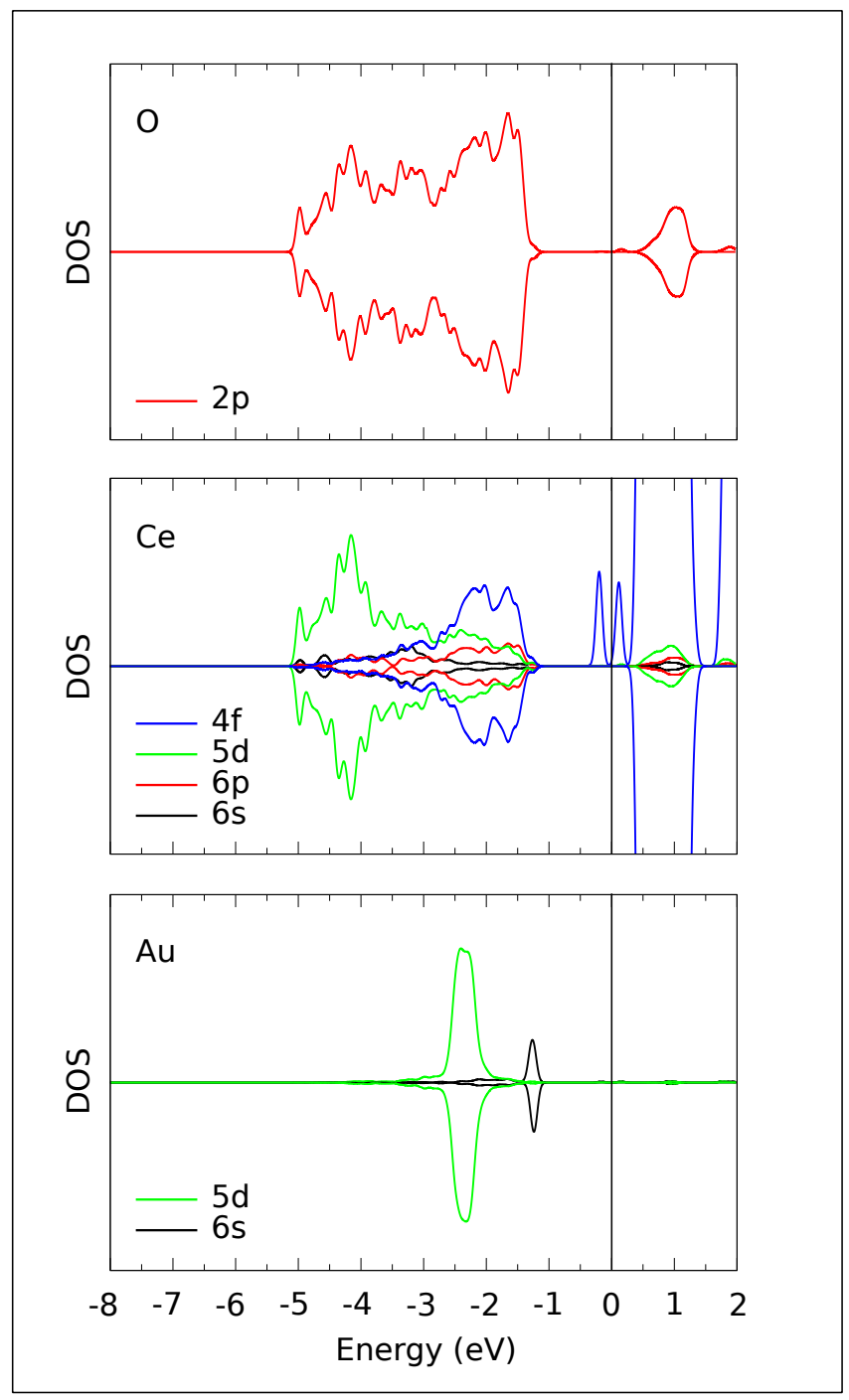

FIG. 8. PDOS for a single $\mathrm{Au}$ adatom adsorbed onto a $\mathrm{CeO}_{2}\{111\}$ surface modified with a single oxygen vacancy. Details of the presentation are as per Fig. 2.

density in Fig. 6.b. This may at first seem a little concerning, but is most likely a consequence of (a) the finite energetic smearing applied when calculating occupancies in our calculations and (b) some slight hybridisation between neighbouring cerium ions mediated via their shared covalent interactions with the intervening oxygen ion. A higher value of $U$, as used in most previous studies, ${ }^{5,8-15}$ would undoubtedly tend to suppress both effects, resulting in less of the spurious spin leakage seen here. In practice, however, the error introduced into our calculated geometry and total energy should be minimal. At any rate, the effect is somewhat smaller in the next case that we shall consider, and all but absent from our calculations of iridium adsorption. 


\section{Au on Oxidised $\mathrm{CeO}_{2}\{111\}$}

Adsorption of gold into a cerium vacancy presents a rather different scenario from the preceding case, since one may reasonably view the resulting situation as an example of metal-atom substitution. In order for the adatom to mimic electronically the oxidation number of the missing cerium cation, however, it would be necessary not only for the Au $6 \mathrm{~s}$ state to be entirely depopulated, but also for three electrons to be removed from the Au $5 d$ states (which would be fully occupied in the isolated atom) and accommodated within the electronic structure of the surrounding material. Unsurprisingly, given the results described above, the situation revealed by the PDOS (Fig. 9) is broadly in line with this surmise, although rather less extreme. The Au $4 f$ states, lying some $80 \mathrm{eV}$ below the Fermi level, are gloriously unaffected upon substitution into the cerium vacancy site, but the Au $5 d$ and $6 s$ states see their occupancies drop by around one and a quarter electrons and one third of an electron respectively, compared with the neutral atom. This seems largely to be associated with the formation of covalently mixed states involving $\mathrm{O}$ $2 p$ contributions, resulting in four spin-symmetric peaks centred at binding energies of $5.00 \mathrm{eV}, 5.50 \mathrm{eV}, 5.75 \mathrm{eV}$ and $6.25 \mathrm{eV}$. The four single-electron peaks observed above the Fermi level on the clean cerium-deficient surface, deriving (it will be recalled) from admixture of $\mathrm{Ce} 4 f$ and $\mathrm{O} 2 p$ states, vanish upon adsorption of the gold adatom, to be replaced with a pair of single-electron peaks either side of the Fermi level having pure $\mathrm{Ce} 4 f$ character. Amidst all of this, the overall occupancy of $\mathrm{O} 2 p$ states rises by about two thirds of an electron, while that of the Ce $5 d$ states falls by about half an electron. Remarkably, however, the cerium and oxygen components of the electronic structure end up looking fairly similar to the situation pertaining to the gold-adsorbed oxygen vacancy, despite the radical differences between the two vacancies in the absence of gold. The major differences between gold adsorption in the cerium and oxygen vacancy sites are, therefore, predominantly limited to the electronic structure of the gold adatom itself, which gains a substantial charge of $+1.51|e|$ on the oxidised surface, compared with the modest negative charge observed on the reduced surface. To place this in context, it should be recalled that the Mulliken charge on cerium ions in the bulk material is just $+1.33|e|$, so the charge acquired by the gold adatom is consistent with a nominal oxidation state of at least +4 .

Spin localisation is rather closer to complete than was the case for adsorption into the oxygen vacancy, with two of the neighbouring cerium ions each gaining a net majority-spin moment of $0.67 \mu_{B}$, associated strongly with their $4 f$ states (see Fig. 6.c). Four of the oxygen ions in the uppermost trilayer acquire small but non-negligible net spin moments in the opposite sense, collectively amounting to $0.27 \mu_{B}$. It would appear that the covalent interaction between $\mathrm{Au}$ $5 d$ and $\mathrm{O} 2 p$ orbitals essentially saturates the unoccupied oxygen states found above the Fermi level in the absence of the adatom, while additional electrons donated from $\mathrm{Au} 5 d$ and $6 s$ states are accommodated on two of the nearby cerium

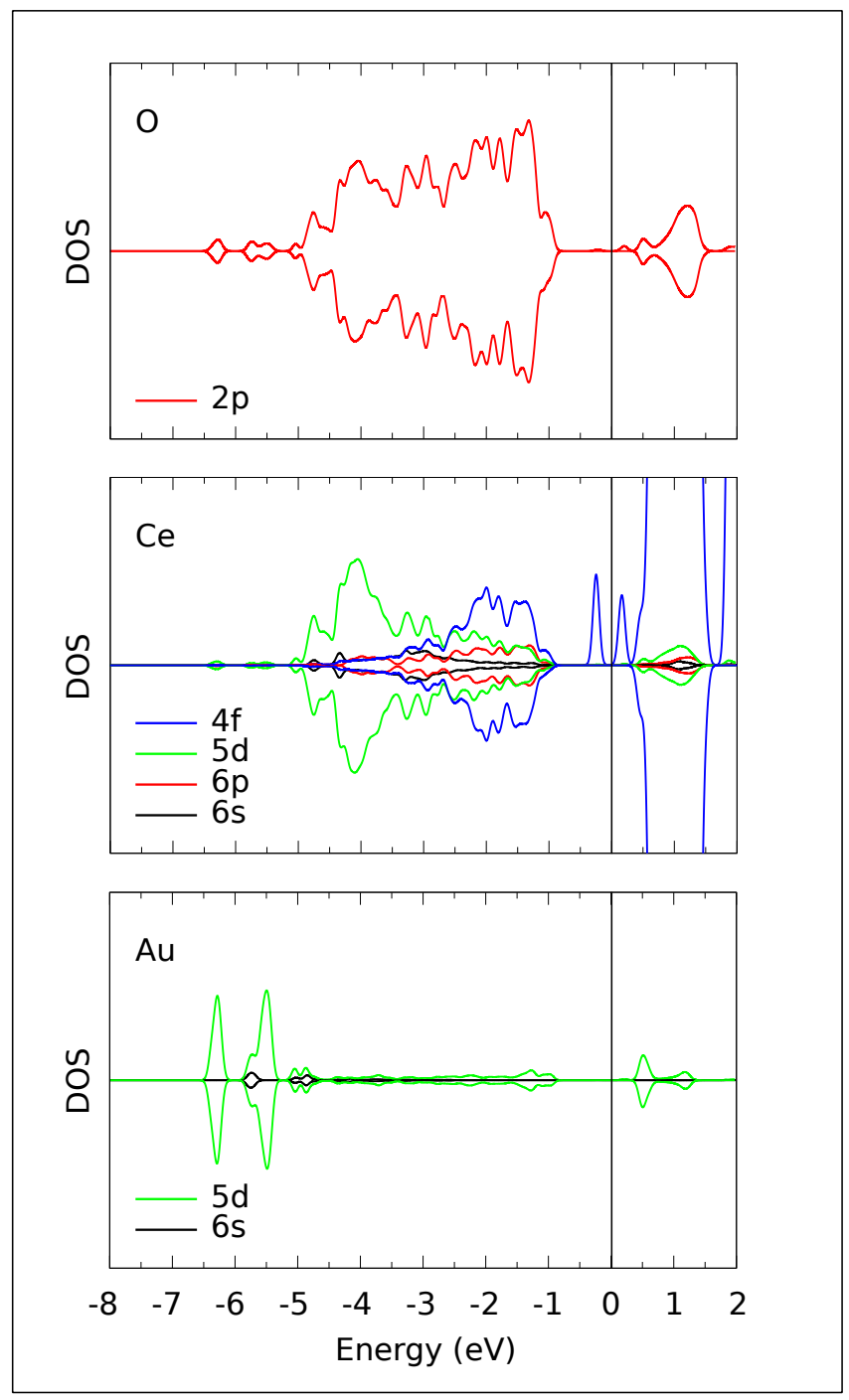

FIG. 9. PDOS for a single $\mathrm{Au}$ adatom adsorbed onto a $\mathrm{CeO}_{2}\{111\}$ surface modified with a single cerium vacancy. Details of the presentation are as per Fig. 2.

ions.

\section{Adsorption of Iridium on $\mathrm{CeO}_{2}\{111\}$ Surfaces}

From a structural perspective, the differences between gold and iridium adsorption on ceria surfaces can largely be explained by the much greater affinity of the latter metal for oxygen (Fig. 10). Where gold adsorbs onto the stoichiometric surface in a bridging geometry between two adjacent top-layer oxygen ions, iridium binds instead at a site midway between three adjacent such ions. Similarly, whereas gold adsorbs directly into the oxygen vacancy site on the reduced surface, we find that iridium binds into an oxygen-bridging geometry, leaning towards the nearby vacancy site but not actually falling into it. Only in the case of an oxidised surface do we 
find the same adsorption geometry for both adspecies, binding directly into the cerium vacancy and hence maximising interaction with oxygen in both scenarios. In terms of stability, we calculate a $\Delta H_{a}$ binding enthalpy of $4.55 \mathrm{eV}$ for iridium on the stoichiometric surface, in reasonable agreement with the value of $4.20 \mathrm{eV}$ calculated by O'Connor et al. ${ }^{16}$ Regarding adsorption onto reduced and oxidised ceria surfaces, for which we are unaware of previous theoretical studies, we calculate $\Delta H_{a}$ binding enthalpies (as per Eqn. 3) of $4.63 \mathrm{eV}$ and $12.78 \mathrm{eV}$ respectively, relative to the respective clean defective surfaces. Including the appropriate formation enthalpies of oxygen and cerium vacancies (as per Eqn. 4) these correspond to $\Delta \widetilde{H}_{a}$ binding enthalpies of $2.31 \mathrm{eV}$ and $7.57 \mathrm{eV}$, relative to the stoichiometric surface.

Following the logic that we previously applied to gold (see above) it will prove useful to compare these latter binding enthalpies with the cohesive energy of iridium, which we calculate to be $7.43 \mathrm{eV}$ per atom (cf. $6.94 \mathrm{eV}$ by experiment). It is immediately apparent, from this comparison, that displacement of cerium by single iridium adatoms will be thermodynamically favoured relative to the existence of large bulk-like iridium clusters, even under redox-neutral conditions. Adsorption of single iridium adatoms adjacent to oxygen vacancies, on the other hand, will only occur if those vacancies are already present, due to oxygen-poor ambient conditions, and even then will be unstable relative to cluster formation. Since we calculate the difference in $\Delta H_{a}$ between adsorption into oxygen vacancies and adsorption onto the stoichiometric surface to be only $0.08 \mathrm{eV}$ (cf. the case of gold, where the corresponding difference was $1.20 \mathrm{eV}$ ) it seems unlikely that kinetic hindrance will play any role in stabilising single-atom iridium on the reduced surface.

\section{Ir on Stoichiometric $\mathrm{CeO}_{2}\{111\}$}

Examining the PDOS corresponding to adsorption of a single iridium adatom on the stoichiometric surface (Fig. 11) we note immediately a number of important differences compared with the case of gold adsorption. Foremost amongst these is that we now see two majority-polarised $\mathrm{Ce} 4 f$ peaks immediately below the Fermi level - one broad, the other sharp - accommodating three and a quarter electrons between them, rather than the single majority-polarised peak accommodating a single electron that was seen upon adsorption of the coinage metal. The broad peak is associated energetically with a like-spin peak in the $\mathrm{O} 2 p$ channel that accommodates roughly three quarters of a electron, so that the overall occupancy of this complex amounts to four electrons in total. As noted in a number of cases above, however, the majority-polarised Ce $4 f$ peaks are assembled primarily from states that already lay below the Fermi level in the absence of the adatom, so that the overall population of this channel increases by only around four fifths of an electron (Table III). A proportion of the electrons needed to fill these states are provided by depletion of previously occupied Ce $5 d$ electrons, with the iridium adatom itself acquiring a charge of $+1.02|e|$.

In terms of spin localisation, all four of the cerium ions ly-
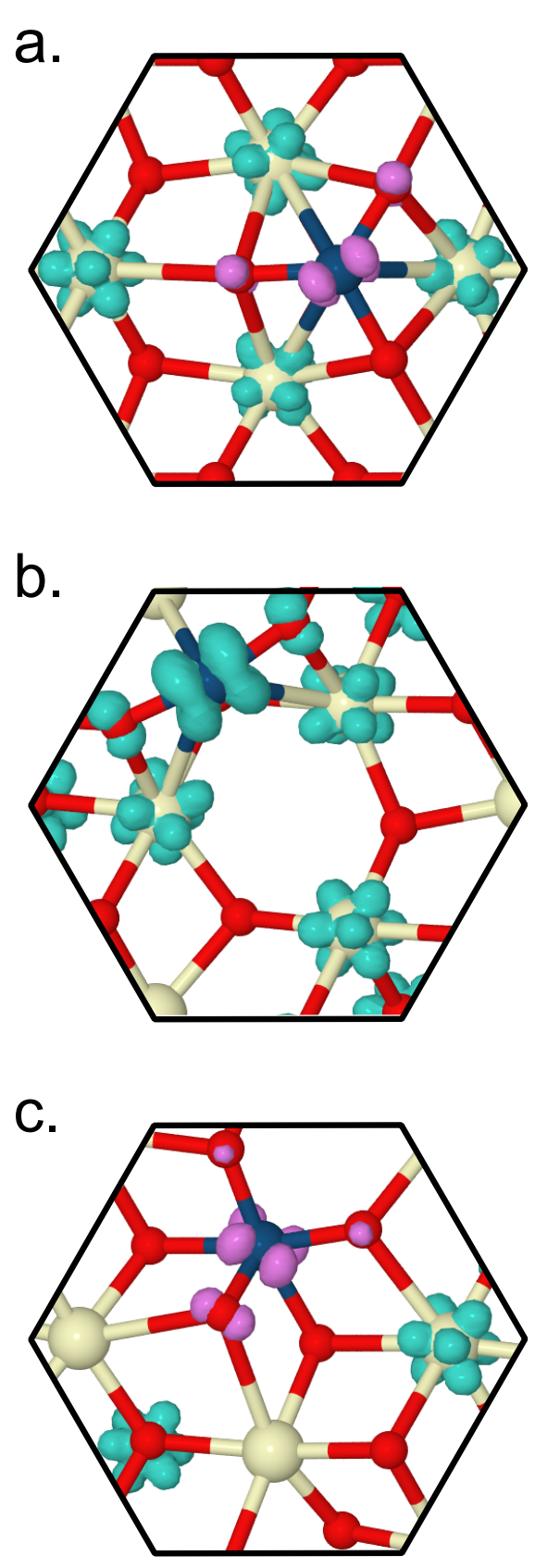

FIG. 10. Orthographic top-down views of an iridium atom adsorbed (a) on the stoichiometric surface, (b) at an oxygen vacancy, and (c) at a cerium vacancy. Details of the presentation are as per Fig. 4. Isosurfaces indicate regions in which the magnitude of the net spin density exceeds $0.1 \mu_{B} . \AA^{-3}$, with turquoise indicating that the overall majority spin species is locally dominant, and lilac indicating that the overall minority spin species is locally dominant.

ing in the uppermost trilayer (within the modelled unit cell) gain similar net majority-spin moments, averaging $0.91 \mu_{B}$, as can be seen readily in Fig. 10.a). Also clearly visible in this image are regions of $p$-like disposition localised on two of the three oxygen ions adjacent to the adatom, associated with net minority-spin moments on these ions amounting to 


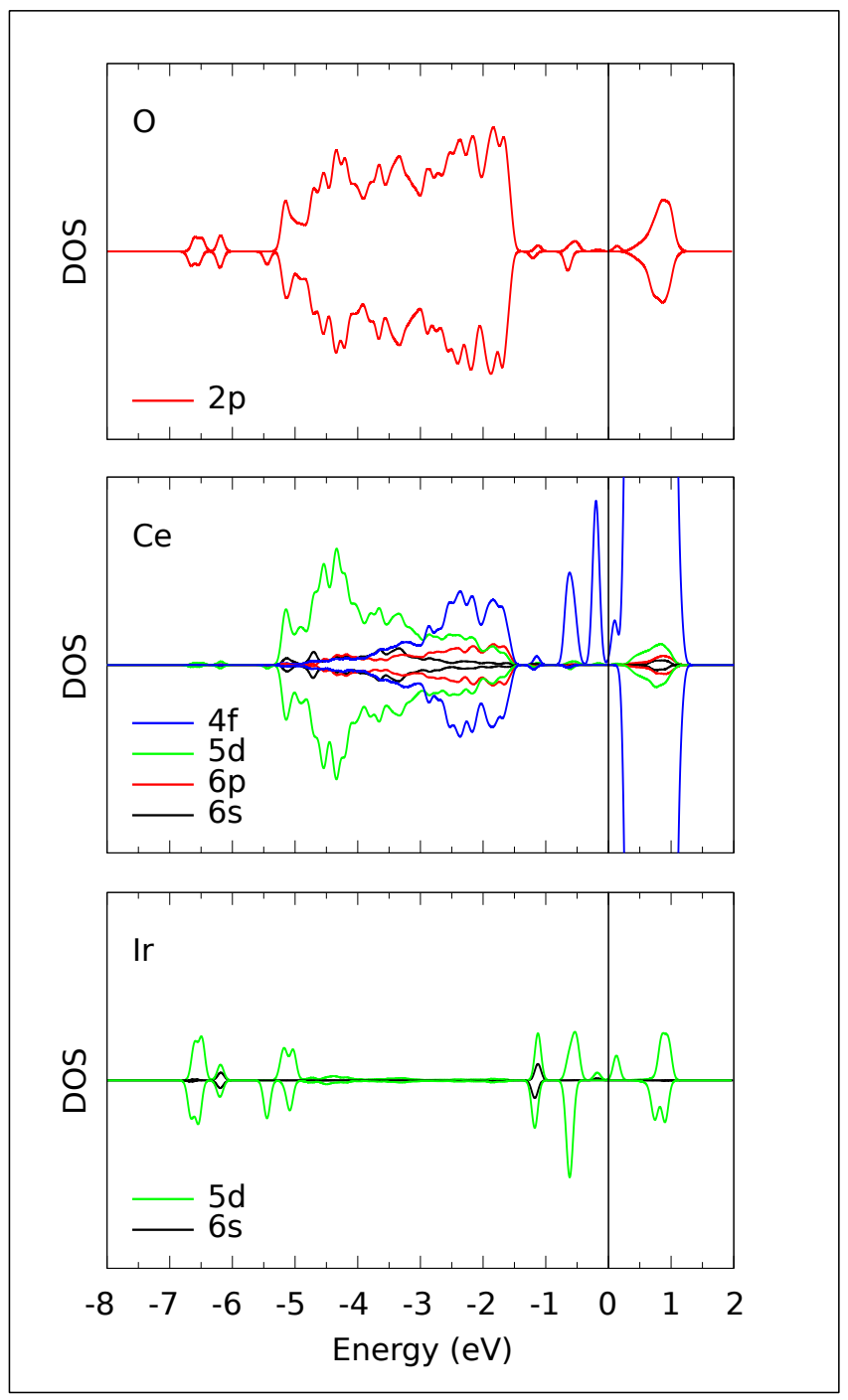

FIG. 11. PDOS for a single Ir adatom adsorbed onto a stoichiometric $\mathrm{CeO}_{2}\{111\}$ surface. Details of the presentation are as per Fig. 2.

$0.10 \mu_{B}$. Similarly apparent is a region of $d$-like disposition located on the iridium adatom, associated with a net minorityspin moment of $0.26 \mu_{B}$. This asymmetry in the spin is reflected in the PDOS, which shows a marked separation in energy between Ir $5 d$ states of majority- and minority-spin character, especially toward the lower edge of ceria's main valence complex and within about $1 \mathrm{eV}$ either side of the Fermi level (Fig. 11). This asymmetry stands in stark contrast to the case of gold, where states belonging to the adatom are found to be essentially spin-symmetric on all three substrates (Figs. 7-9). Nevertheless, despite the presence of Ir $5 d$ peaks immediately above and below the Fermi level, the density of states at the Fermi level itself remains strictly zero.

\section{Ir on Reduced $\mathrm{CeO}_{2}\{111\}$}

Turning to the situation where iridium adsorbs in an oxygen-bridging geometry adjacent to an oxygen vacancy, the electronic structure of the substrate turns out to look surprisingly similar to the stoichiometric case we have just discussed. Specifically, three majority-polarised Ce $4 f$ peaks - one tall, two shorter - are visible in the PDOS (Fig. 12) immediately below the Fermi level, accommodating around three and two thirds electrons in all, roughly coincident in energy with a small peak of $\mathrm{O} 2 p$ character that accommodates a further one third of an electron. As in the stoichiometric case, therefore, we must account for a total of four unpaired electrons associated with this complex. Examination of spin localisation reveals that two of the three cerium ions lying closest to the adatom acquire net majority-spin moments of $0.97 \mu_{B}$, while the more distant cerium ion within the uppermost trilayer gains a net majority-spin moment of $1.06 \mu_{B}$ and a single cerium atom in the underlying trilayer gains a net majority-spin moment of $0.88 \mu_{B}$. These observations are, once again, consistent with visualisation of the net spin density (Fig. 10.b). Moreover, it may be that the O $2 p$ contribution to this complex accounts for the net majority-spin moment of $0.19 \mu_{B}$ associated with each of the two oxygen ions bridged by the adatom (and similarly apparent in the net spin visualisation).

This superficial similarity in electronic structure between the stoichiometric and oxygen-vacancy cases for iridium adsorption does not, however, extend to the adatom itself, which gains a charge when binding adjacent to the oxygen vacancy of only $+0.53|e|-$ barely half of its acquired charge when binding on the stoichiometric surface. On the other hand, the adatom gains a net majority-spin moment of $0.86 \mu_{B}$ when adsorbed adjacent to the oxygen vacancy, which is more than three times greater than on the stoichiometric surface, and aligned with the overall majority-spin sense rather than opposed to it. Mulliken analysis confirms that this spin asymmetry is associated exclusively with changes in Ir $5 d$ occupancy, entirely consistent with visualisation of the net spin density (Fig. 10.b). The associated spin-asymmetry observable in the projected Ir $5 d$ states (Fig. 12) is arguably more substantial than when adsorbed on the stoichiometric surface, and is accompanied by a marked spin-asymmetry in the projected $\operatorname{Ir} 6 s$ states, which was not the case in the stoichiometric system. Furthermore, there is a non-vanishing Ir $5 d$ density of states at the Fermi level, amounting to approximately $1 \mathrm{eV}^{-1}$ per adatom and entirely of majority-spin character.

\section{Ir on Oxidised $\mathrm{CeO}_{2}\{111\}$}

At last, then, we come to consider the electronic structure corresponding to adsorption of an iridium adatom into a cerium vacancy. It will be recalled that this is the only case where the geometry is comparable with that adopted upon adsorption of a gold adatom, with the adsorbate occupying the cerium vacancy itself in both instances. Unsurprisingly, perhaps, there are also some notable similarities to be found 


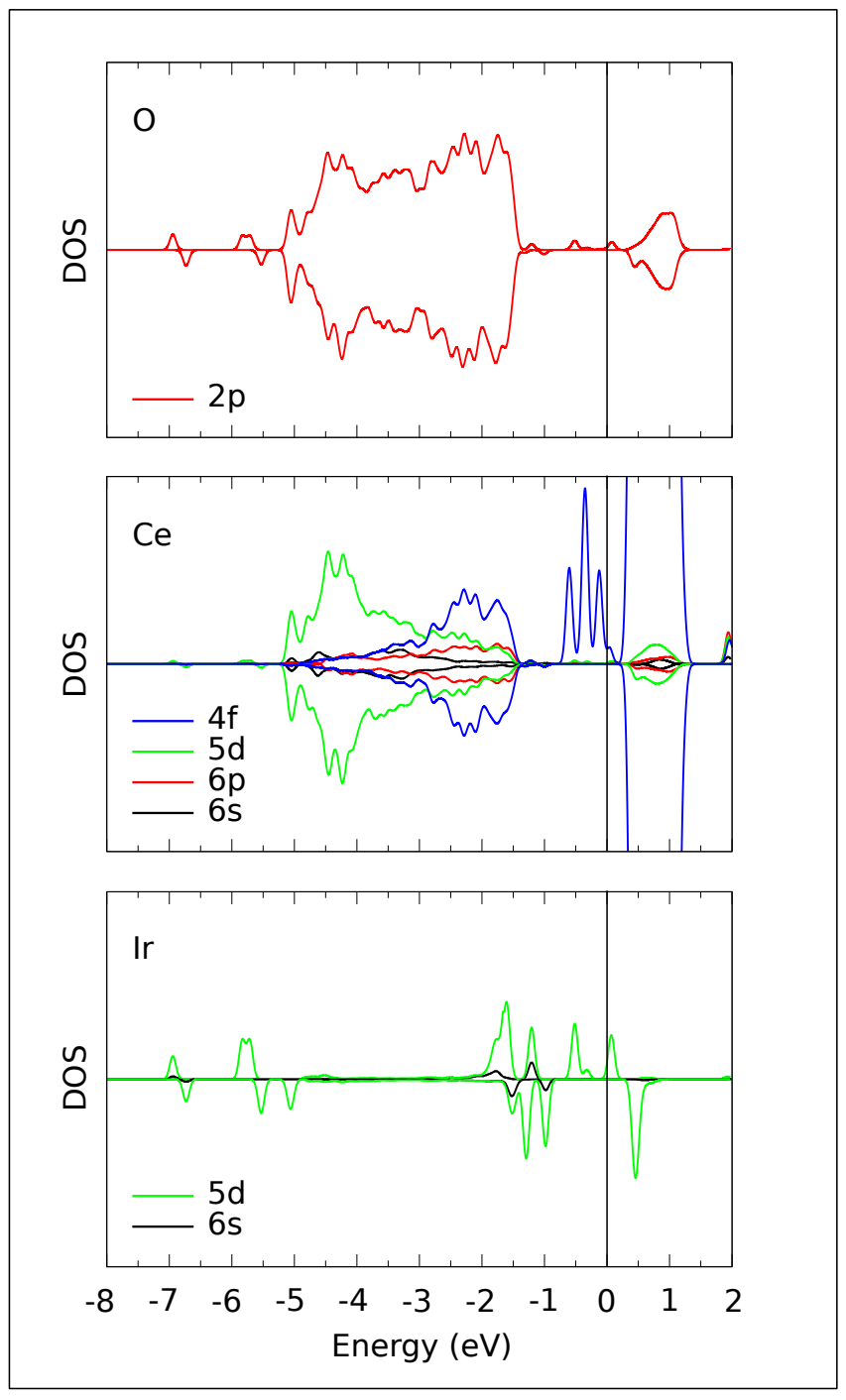

FIG. 12. PDOS for a single Ir adatom adsorbed onto a $\mathrm{CeO}_{2}\{111\}$ surface modified with a single oxygen vacancy. Details of the presentation are as per Fig. 2.

between the gold-adsorbed and iridium-adsorbed electronic structures. For example, we note the appearance of five almost spin-symmetric $\mathrm{O} 2 p$ peaks in the binding-energy range 5.30$6.50 \mathrm{eV}$ (accommodating roughly six electrons) clearly associated with a cluster of almost spin-symmetric Ir $5 d$ peaks (accommodating about four electrons) that appear at very nearly the same energies (Fig. 13). As in the case of gold adsorption, the four unoccupied peaks associated with $\mathrm{O} 2 p$ states in the absence of an adatom vanish, and it is hard to escape the conclusion that covalent bonding between the iridium adatom and its neighbouring oxygen atoms is the proximal cause. The cases are not precisely alike, of course, since the adsorption of gold generates just four spin-symmetric occupied peaks, where iridium generates five, but the broad similarity is striking. Indeed, the increase in population of the $\mathrm{O} 2 p$ states upon adsorption is almost precisely the same here as it was for the case of a gold adatom, and the rather minor effect upon popu- lation of the $\mathrm{O} 2 s$ states also differs only somewhat (Table III).

Regarding the distribution of electrons between cerium ions upon iridium adsorption, this superficially resembles the situation described above for gold adsorption into a cerium vacancy, with non-negligible spin moments found on just two of their number. Looking in a little more detail, however, we note that where both such ions were located within the uppermost trilayer when the adatom was gold, here this is true for only one of the high-spin ions, having a net majority-spin moment of $0.93 \mu_{B}$, the other lying in the second trilayer, with a net majority-spin moment of $0.70 \mu_{B}$ (Fig. 10.c). This distribution is presumably attributable to the two majority-polarised single-electron peaks visible in the Ce $4 f$ spectrum close to the Fermi level (Fig. 13). In fact, in contrast to all other systems discussed in the present work, we find that one of these peaks actually straddles the Fermi level, so that the combined occupancy of the pair amounts to very nearly one and a half electrons. The corresponding Ce $4 f$ density of states at the Fermi level is around $7.5 \mathrm{eV}^{-1}$ per adsorbed iridium atom.

A further apparent similarity between the adsorption of gold and iridium into the cerium vacancy also turns out to be a little deceptive. Both cases share the distinction of resulting in the largest charge accumulation on the adatom, amounting to $+1.51|e|$ for gold (it will be recalled) and $+1.74|e|$ for iridium. Closer examination of our Mulliken populations reveals, however, that whilst the majority of the electrons donated by gold (c.81\%) come from its $5 d$ states, the majority of those donated by iridium (c.84\%) come from its $6 \mathrm{~s}$ state (Table III). Furthermore, where the gold adatom remains spin-compensated in all circumstances, the iridium adatom acquires a net minority-spin moment of $0.29 \mu_{B}$ when adsorbed into the cerium vacancy, which is rather similar in both sign and magnitude to its disposition on the stoichiometric surface. Once again, visualisation of the net spin density (Fig. 10.c) makes it clear, alongside our Mulliken analysis, that this spinasymmetry is associated exclusively with the Ir $5 d$ states. Spin asymmetry is also apparent in the PDOS (Fig. 13) where it is particularly noteworthy that the Fermi level coincides precisely with the peak of a majority-polarised Ir $5 d$ peak. As a result, the Ir $5 d$ density of states at the Fermi level approximates $2 \mathrm{eV}^{-1}$ per adatom, roughly double that noted above for adsorption of iridium into an oxygen vacancy. For comparison, we calculate the Fermi-level density of states in bulk iridium to be close to $1.5 \mathrm{eV}^{-1}$ per atom.

\section{CONCLUSIONS}

The present work has highlighted a number of important differences between the adsorption of single-atom iridium and single-atom gold on the $\mathrm{CeO}_{2}\{111\}$ surface. From a structural perspective, the transition metal displays a markedly stronger affinity for binding to the oxygen ions present in the uppermost trilayer, adopting geometries that maximise covalent interaction to the greatest achievable degree. On the stoichiometric surface, iridium settles into a site permitting binding to three neighbouring oxygen ions, for example, whereas gold favours a site that merely bridges between two such ions. In 


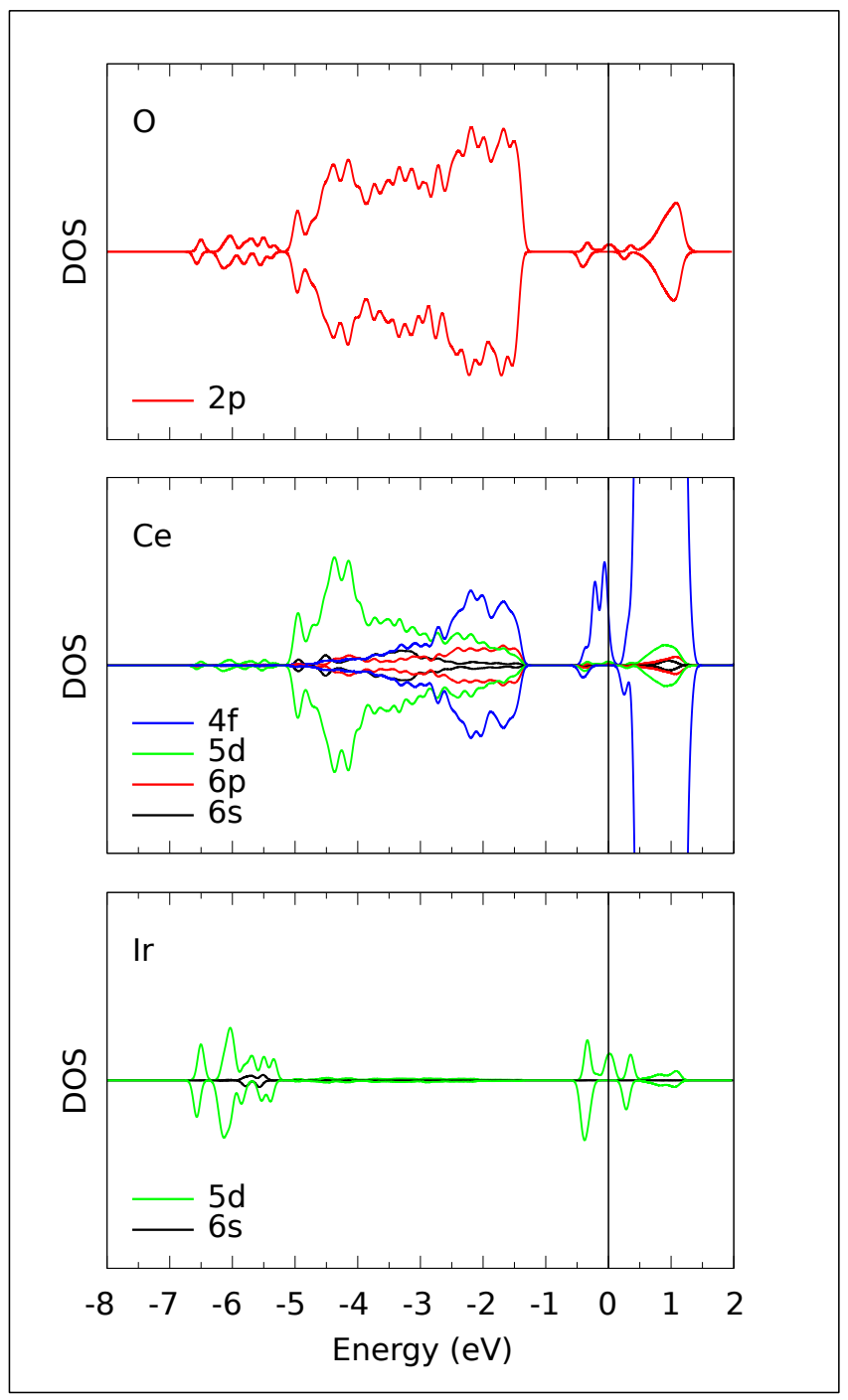

FIG. 13. PDOS for a single Ir adatom adsorbed onto a $\mathrm{CeO}_{2}\{111\}$ surface modified with a single cerium vacancy. Details of the presentation are as per Fig. 2.

the presence of an oxygen vacancy, on the other hand, iridium binds to two nearby oxygen ions, whereas gold settles into the vacancy itself and interacts predominantly with the surrounding cerium ions. Only for surfaces displaying a cerium vacancy do we find a similar adsorption geometry for both species, substituting directly for the missing metal atom in each case.

From the energetic perspective, it is hardly surprising that iridium, as the more reactive species, binds more strongly in every scenario than does gold in the equivalent situation. In particular, it is noteworthy that the less reactive coinage metal does not appear to be stable in single-atom form under redoxneutral conditions. Only under sufficiently oxidising conditions that cerium vacancies become prevalent will single-atom gold become thermodynamically stable, whilst stability under reducing or redox-neutral conditions can only be explained in terms of kinetic resistance to sintering conferred by the rel- ative stability of adsorption into oxygen vacancy sites compared with adsorption onto the stoichiometric surface. For the transition metal, on the other hand, we find that even under redox-neutral conditions one can expect displacement of individual cerium ions by single iridium atoms, suggesting that the substitutional geometry is the most promising candidate for catalytic stability.

Finally, from the perspective of electronic structure there are two primary differences between systems involving the adsorption of single-atom gold and those involving the adsorption of single-atom iridium. In the first place, the influence of the adsorbate upon the substrate is significantly more profound in the case of the transition metal; more majority-polarised $\mathrm{Ce} 4 f$ peaks accumulate in the vicinity of the Fermi level within all three iridium-adsorbed scenarios (stoichiometric, reduced, and oxidised) than for the corresponding gold-adsorbed cases, and in the instance where iridium adsorbs into a cerium vacancy these even result in a sizeable density of states at the Fermi level itself. Secondly, electronic states localised on the iridium adatom develop a substantial degree of spin-asymmetry that is absent from the gold adatom, resulting in polarisation of the Ir $5 d$ states and a further contribution to the non-zero density of states apparent at the Fermi level. Collectively, these differences ought to imply not only that single-atom iridium should prove more reactive toward adsorbing molecular species, but also that the surrounding oxide support may itself be expected to play a more active role in catalysis than might be the case when host to single-atom gold. Ultimately, the veracity of such statements will need to be tested with reference to specific catalytic targets, for example methane activation, but further investigation of such matters we defer to a future publication.

\section{DATA AVAILABILITY}

The data that supports the findings of this study are available within the article [and its supplementary material].

\section{SUPPLEMENTARY MATERIAL}

See supplementary material for details concerning the choice of Hubbard parameter, and for output files from the reported calculations.

\section{ACKNOWLEDGMENTS}

Calculations were performed at the University of Cambridge High Performance Computing Service. CJO would like to acknowledge the Winston Churchill Foundation of the United States for funding a graduate scholarship. 
${ }^{1}$ Z. W. Chen, L. X. Chen, C. C. Yang, and Q. Jiang, "Atomic (single, double, and triple atoms) catalysis: frontiers, opportunities, and challenges," J. Mater. Chem. A 7, 3492-3515 (2019).

${ }^{2}$ S. K. Kaiser, Z. Chen, D. Faust Akl, S. Mitchell, and J. Pérez-Ramírez, "Single-atom catalysts across the periodic table," Chem. Rev. 120, 11703 11809 (2020).

${ }^{3}$ B. Qiao, A. Wang, X. Yang, L. F. Allard, Z. Jiang, Y. Cui, J. Liu, J. Li, and T. Zhang, "Single-atom catalysis of $\mathrm{CO}$ oxidation using $\mathrm{Pt}_{1} / \mathrm{FeO}_{x}$," Nat. Chem. 3, 634-641 (2011)

${ }^{4}$ Q. Fu, H. Saltsburg, and M. Flytzani-Stephanopoulos, "Active nonmetallic $\mathrm{Au}$ and Pt species on ceria-based water-gas shift catalysts," Science 301, 935-938 (2003).

${ }^{5}$ D. Tibiletti, A. Amieiro-Fonseca, R. Burch, Y. Chen, J. M. Fisher, A. Goguet, C. Hardacre, P. Hu, and D. Thompsett, "DFT and in situ EXAFS investigation of gold/ceria-zirconia low-temperature water gas shift catalysts: identification of the nature of the active form of gold," J. Phys. Chem. B 109, 22553-22559 (2005).

${ }^{6}$ Z. P. Liu, S. J. Jenkins, and D. A. King, "Origin and activity of oxidized gold in water-gas-shift catalysis," Phys. Rev. Lett. 94, 196102 (2005).

${ }^{7}$ V. Shapovalov and H. Metiu, "Catalysis by doped oxides: $\mathrm{CO}$ oxidation by $\mathrm{Au}_{x} \mathrm{Ce}_{1-x} \mathrm{O}_{2}$," J. Catal. 245, 205-214 (2007).

${ }^{8}$ C. Zhang, A. Michaelides, D. A. King, and S. J. Jenkins, "Structure of gold atoms on stoichiometric and defective ceria surfaces," J. Chem. Phys. 129, 194708 (2008).

${ }^{9}$ N. C. Hernández, R. Grau-Crespo, N. H. de Leeuw, and J. F. Sanz, "Electronic charge transfer between ceria surfaces and gold adatoms: a GGA + $U$ investigation," Phys. Chem. Chem. Phys. 11, 5246-5252 (2009).

${ }^{10} \mathrm{M}$. F. Camellone and S. Fabris, "Reaction mechanisms for the co oxidation on $\mathrm{Au} / \mathrm{CeO}_{2}$ catalysts: activity of substitutional $\mathrm{Au}^{3+} / \mathrm{Au}^{+}$cations and deactivation of $\mathrm{Au}^{+}$adatoms," J. Am. Chem. Soc. 131, 10473-10483 (2009).

${ }^{11}$ C. Zhang, A. Michaelides, D. A. King, and S. J. Jenkins, "Anchoring sites for initial Au nucleation on $\mathrm{CeO}_{2}\{111\}$ : O vacancy versus Ce vacancy," J. Phys. Chem. C 113, 6411-6417 (2009).

${ }^{12}$ C. Zhang, A. Michaelides, D. A. King, and S. J. Jenkins, "Positive charge states and possible polymorphism of gold nanoclusters on reduced ceria," J. Am. Chem. Soc. 132, 2175-2182 (2010).

${ }^{13}$ C. Zhang, A. Michaelides, and S. J. Jenkins, "Theory of gold on ceria," Phys. Chem. Chem. Phys. 13, 22-33 (2011).

${ }^{14}$ B. T. Teng, F. M. Wu, W. X. Huang, X. D. Wen, L. H. Zhao, and M. F. Luo, "A DFT study of the structures of $\mathrm{Au}_{x}$ clusters on a $\mathrm{CeO}_{2}(111)$ surface," ChemPhysChem 13, 1261-1271 (2012).

${ }^{15}$ J. Engel, E. Schwartz, C. R. A. Catlow, and A. Roldan, "The influence of oxygen vacancy and $\mathrm{Ce}^{3+}$ ion positions on the properties of small gold clusters supported on $\mathrm{CeO}_{2-x}(111)$," J. Mater. Chem. A 8, 15695-15705 (2020).

${ }^{16}$ N. J. O'Connor, A. S. M. Jonayat, M. J. Janik, and T. P. Senftle, “Interaction trends between single metal atoms and oxide supports identified with density functional theory and statistical learning," Nature Catalysis 1, 531-539 (2018).

${ }^{17}$ M. A. van Spronsen, K. Daunmu, C. R. O'Connor, T. Egle, H. Kersell, J. Oliver-Meseguer, M. B. Salmeron, R. J. Madix, P. Sautet, and C. M. Friend, "Dynamics of surface alloys: Rearrangement of $\mathrm{Pd} / \mathrm{Ag}(111)$ induced by $\mathrm{CO}$ and $\mathrm{O}_{2}$," J. Phys. Chem. C 123, 8312-8323 (2019).

${ }^{18} \mathrm{~F}$. Li, Y. Li, X. C. Zeng, and Z. Chen, "Exploration of high-performance single-atom catalysts on support $\mathrm{M}_{1} / \mathrm{FeO}_{x}$ for $\mathrm{CO}$ oxidation via computational study," ACS Catalysis 5, 544-552 (2015).

${ }^{19}$ G. S. Parkinson, "Single-atom catalysis: How structure influences catalytic performance," Catal. Lett. 149, 1137-1146 (2019).

${ }^{20}$ S. Dey and G. C. Dhal, "Cerium catalysts applications in carbon monoxide oxidations," Mater. Sci. Energy Technol. 3, 6-24 (2020).

${ }^{21}$ C. T. Campbell and J. R. V. Sellers, "Anchored metal nanoparticles: Effects of support and size on their energy, sintering resistance and reactivity," Faraday Discuss. 162, 9-30 (2013).

${ }^{22} \mathrm{C}$. T. Campbell, "The energetics of supported metal nanoparticles: Relationships to sintering rates and catalytic activity," Accounts of Chemical Research 46, 1712-1719 (2013).

${ }^{23}$ M. Nolan, S. Grigoleit, D. C. Sayle, S. C. Parker, and G. W. Watson, "Density functional theory studies of the structure and electronic structure of pure and defective low index surfaces of ceria," Surf. Sci. 576, 217-229 (2005).
${ }^{24}$ D. D. Koelling, A. M. Boring, and J. H. Wood, "The electronic structure of $\mathrm{CeO}_{2}$ and $\mathrm{PrO}_{2}$," Solid State Commun. 47, 227-232 (1983).

${ }^{25}$ E. Wuilloud, B. Delley, W. D. Schneider, and Y. Baer, "Spectroscopic evidence for localized and extended $f$-symmetry states in $\mathrm{CeO}_{2}$," Phys. Rev. Lett. 53, 202-205 (1984).

${ }^{26}$ A. Pfau and K. D. Schierbaum, "The electronic structure of stoichiometric and reduced $\mathrm{CeO}_{2}$ surfaces: an XPS, UPS and HREELS study," Surf. Sci. 321, 71-80 (1994).

${ }^{27}$ M. V. Ganduglia-Pirovano, A. Hofmann, and J. Sauer, “Oxygen vacancies in transition metal and rare earth oxides: Current state of understanding and remaining challenges," Surf. Sci. Rep. 62, 219-270 (2007).

${ }^{28}$ R. K. Grasselli and J. D. Burrington, "Selective oxidation and ammoxidation of propylene by heterogeneous catalysis," (Academic Press, 1981) pp. 133-163.

${ }^{29}$ H.-Y. Li, H.-F. Wang, X.-Q. Gong, Y.-L. Guo, Y. Guo, G. Lu, and P. Hu, "Multiple configurations of the two excess $4 f$ electrons on defective $\mathrm{CeO}_{2}$ (111): Origin and implications," Phys. Rev. B 79, 193401 (2009).

${ }^{30}$ M. V. Ganduglia-Pirovano, J. L. F. Da Silva, and J. Sauer, "Densityfunctional calculations of the structure of near-surface oxygen vacancies and electron localization on $\mathrm{CeO}_{2}(111)$," Phys. Rev. Lett. 102, 026101 (2009).

${ }^{31} \mathrm{H}$. Norenberg and G. A. D. Briggs, "Defect structure of nonstoichiometric $\mathrm{CeO}_{2}$ (111) surfaces studied by scanning tunneling microscopy," Phys. Rev. Lett. 79, 4222 (1997).

${ }^{32}$ Y. Namai, K. Fukui, and Y. Iwasawa, "Atom-resolved noncontact atomic force microscopic observations of $\mathrm{CeO}_{2}(111)$ surface with different oxidation states: Surface structure and behaviour of surface oxygen atoms," J. Phys. Chem. B 107, 11666 (2003).

${ }^{33}$ F. Esch, S. Fabris, L. Zhou, T. Montini, C. Africh, P. Fornasiero, G. Comelli, and R. Rosei, "Electron localization determines defect formation on ceria substrates," Science 309, 752-755 (2005).

${ }^{34}$ C. Zhang, A. Michaelides, D. A. King, and S. J. Jenkins, "Oxygen vacancy clusters on ceria: Decisive role of cerium $f$ electrons," Phys. Rev. B 79, 075433 (2009).

${ }^{35}$ N. S. Shuman, S. G. Ard, B. C. Sweeny, H. Pan, A. A. Viggiano, N. R. Keyes, H. Guo, C. J. Owen, and P. B. Armentrout, " $\mathrm{Au}_{2}^{+}$cannot catalyze conversion of methane to ethene at low temperature," Catal. Sci. Technol. 9, 2767-2780 (2019).

${ }^{36}$ P. B. Armentrout, B. C. Stevenson, F. Yang, F. J. Wensink, O. V. Lushchikova, and J. M. Bakker, "Infrared spectroscopy of gold carbene cation $\left(\mathrm{AuCH}_{2}^{+}\right)$: Covalent or dative bonding?" J. Phys. Chem. A 123, 8932-8941 (2019).

${ }^{37}$ Z. Liang, T. Li, M. Kim, A. Asthagiri, and J. F. Weaver, "Low-temperature activation of methane on the $\mathrm{IrO}_{2}(110)$ surface," Science 356, 299-303 (2017).

${ }^{38}$ S. J. Clarke, M. D. Segall, C. J. Pickard, P. J. Hasnip, M. J. Probert, K. Refson, and M. C. Payne, "First-principles methods using CASTEP," Z. Kristallogr. 220, 567-570 (2005).

${ }^{39}$ H. J. Monkhorst and J. D. Pack, "Special points for brillouin-zone integrations," Phys. Rev. B 13, 5188-5192 (1976).

${ }^{40}$ N. Marzari, D. Vanderbilt, and M. C. Payne, "Ensemble density functional theory for ab initio molecular dynamics of metals and finite-temperature insulators," Phys. Rev. Lett. 79, 1337-1340 (1997).

${ }^{41} \mathrm{D}$. Vanderbilt, "Soft self-consistent pseudopotentials in a generalized eigenvalue formalism," Phys. Rev. B 41, 7892-7895 (1990).

${ }^{42}$ J. P. Perdew, K. Burke, and M. Ernzerhof, "Generalized gradient approximation made simple," Phys. Rev. Lett. 77, 3865-3868 (1996).

${ }^{43}$ B. Hammer, L. B. Hansen, and J. K. Norskov, "Improved adsorption energetics within density-functional theory using revised Perdew-BurkeErnzerhof functionals," Phys. Rev. B 59, 7413-7421 (1999).

${ }^{44}$ R. Fletcher, Practical Methods of Optimisation, 2nd Ed. (John Wiley \& Sons, 1987).

${ }^{45}$ C. Loschen, J. Carrasco, K. M. Neyman, and F. Illas, "First-principles LDA $+\mathrm{U}$ and GGA + U study of cerium oxides: dependence on the effective $\mathrm{U}$ parameter," Phys. Rev. B 75, 035115 (2007).

${ }^{46}$ J. P. Perdew, J. A. Chevary, S. H. Vosko, K. A. Jackson, M. R. Pederson, D. J. Singh, and C. Fiolhais, "Atoms, molecules, solids, and surfaces: Applications of the generalized gradient approximation for exchange and correlation,” Phys. Rev. B 46, 6671-6687 (1992). 
${ }^{47}$ R. S. Mulliken, "Electronic population analysis on LCAO-MO molecular wave functions. I," J. Chem. Phys. 23, 1833-1840 (1955). 